\title{
直觉模糊集和区间值模糊集的截集、分解定理和表现 定理
}

袁学海*, 李洪兴, 孙凯彪

大连理工大学电子与信息工程学院, 大连 116024

*通信作者 E-mail: yuanxuehai@yahoo.com.cn

收稿日期: 2008-06-05; 接受日期: 2008-10-05

国家自然科学基金资助项目 (批准号: 60774049)

摘要该文给出了直觉模糊集和区间值模糊集的截集定义, 建立了直觉模糊集和区 间值模糊集的分解定理和表现定理. 首先, 将直觉模糊集的截集视为三值模糊集, 给出 了直觉模糊集的四类截集定义, 指出这些截集是模糊集截集概念的推广且与模糊集的 截集有完全一样的性质. 其次, 利用本文给出的截集概念, 建立了直觉模糊集的分解定 理和表现定理. 指出直觉模糊集的每种截集都对应两种分解定理和表现定理, 从而建 立了直觉模糊集的八种分解定理和八种表现定理. 最后, 利用直觉模糊集的截集理论, 给出了区间值模糊集的截集定义, 并建立了区间值模糊集的八种分解定理和表现定理. 这些工作为研究直觉模糊集和区间值模糊集建立了理论基础.

\section{关键词}

直觉模糊集

区间值模糊集

三值模糊集

截集

分解定理

表现定理

\section{1 引言}

自从 Zadeh ${ }^{[1]}$ 引入模糊集的概念以来, 模糊集和 系统理论得到了快速的发展. 众所周知, 在模糊系统 理论的研究中, 截集是一个非常重要的概念, 截集在 模糊拓扑 $[2,3]$ 、模糊代数 $[4,5]$ 、模糊测度与模糊分析 [6 10]、模糊优化与决策 ${ }^{[11,12]}$ 、模糊推理 ${ }^{[13,14]}$ 和模 糊逻辑 ${ }^{[15]}$ 等领域都发挥着重要的作用. 因为截集是 联系模糊集合与经典集合的桥梁. 有了截集的概念, 才能建立分解定理和表现定理 ${ }^{[16]}$. 文献 [17] 利用模 糊点与模糊集的邻属关系刻画了模糊集的截集, 指出 模糊集的截集可有四种定义, 每种截集都有类似的性 质且基于每种截集都可建立模糊集的分解定理和表 现定理.

随着模糊集理论的发展, 各种 $L$ - 模糊集相继 出现, 其中最常用的是直觉模糊集 ${ }^{[18]}$ 和区间值模 糊集 [19]. 尽管直觉模糊集和区间值模糊集是等价 的 ${ }^{[20,21]}$. 但人们根据需要仍然从不同角度对这两个
模糊系统进行研究. 文献 [22] 给出了直觉模糊集截 集的定义, 文献 [23 25] 给出了区间值模糊集截集的 定义, 并建立了区间值模糊集的分解定理和表现定 理. 这些研究在相应的模糊系统中都发挥着积极的 作用.

但是通过分析不难发现, 文献 $[22,23]$ 定义的截集 都是用 $[0,1]$ 上的区间数 $\left[\lambda_{1}, \lambda_{2}\right]$ (或 $[0,1]$ 中的两个数 $\left.\left(\lambda_{1}, \lambda_{2}\right), \lambda_{1}+\lambda_{2} \leqslant 1\right)$ 与区间值 (或直觉) 模糊集的隶 属度 (也是 $[0,1]$ 上的区间数或 $[0,1]$ 中的两个数) 去 比较. 这相当于两个二维向量之间进行比较. 由于区 间数之间的序关系不是全序, 因此正如本文第三节中 将要指出的那样, 这种截集不满足文献 [16] 总结的模 糊集截集的性质 5 和性质 6 , 文献 [23] 给出的 $(2,2)$ 截集不保持 “并” 运算, 以此建立的表现定理也不保 持 “余” 运算. 因此, 为了更好的研究直觉模糊集和 区间值模糊集, 有必要给出更理想的截集的定义.

本文突破了“截集必须是经典集合” 的限制, 仍 用 $[0,1]$ 中的数 $\lambda$ 去截直觉模糊集和区间值模糊集, 
从而将直觉 (或区间值) 模糊集的截集定义为三值模 糊集.

本文的结构如下: 第二节介绍本文需要的预备知 识; 第三节在分析文献 [22 24] 定义的区间值模糊集 截集的不足的基础上, 给出了直觉模糊集的四种截集 定义, 研究了这些截集的性质; 在第四节和第五节, 利 用我们给出的截集定义建立了直觉模糊集的八种分 解定理和八种表现定理; 在第六节, 利用直觉模糊集 截集的理论导出了区间值模糊集的截集定义,并建立 了区间值模糊集的分解定理和表现定理.

\section{2 预备知识}

定义 $2.1^{[1]}$ 设 $X$ 为集合, 称映射 $A: X \longrightarrow$ $[0,1]$ 为 $X$ 的一个模糊子集.

定义 $2.2^{[16,17]}$ 设 $A$ 为集合 $X$ 的一个模糊子 集, $\lambda \in[0,1]$, 则称

$$
\begin{aligned}
& A_{\lambda}=\{x \mid x \in X, A(x) \geqslant \lambda\}, \\
& A_{\underline{\lambda}}=\{x \mid x \in X, A(x)>\lambda\},
\end{aligned}
$$

分别为 $A$ 的 $\lambda$ - 上截集和 $\lambda$ - 强上截集.

称

$$
\begin{aligned}
& A^{\lambda}=\{x \mid x \in X, A(x) \leqslant \lambda\}, \\
& A^{\underline{\lambda}}=\{x \mid x \in X, A(x)<\lambda\},
\end{aligned}
$$

分别为 $A$ 的 $\lambda$-下截集和 $\lambda$ - 强下截集.

称

$$
\begin{aligned}
& A_{[\lambda]}=\{x \mid x \in X, A(x)+\lambda \geqslant 1\}, \\
& A_{[\underline{\lambda}]}=\{x \mid x \in X, A(x)+\lambda>1\},
\end{aligned}
$$

分别为 $A$ 的 $\lambda$ - 上重截集和 $\lambda$ - 强上重截集.

称

$$
\begin{aligned}
& A^{[\lambda]}=\{x \mid x \in X, A(x)+\lambda \leqslant 1\}, \\
& A^{[\lambda]}=\{x \mid x \in X, A(x)+\lambda<1\},
\end{aligned}
$$

分别为 $A$ 的 $\lambda$-下重截集和 $\lambda$ - 强下重截集.

定义 $2.3^{[2]}$ 若 $L$ 是一个带有逆合对应的完全 分配格, 则称 $L$ 是一个 $F$ 格.

定义 $\mathbf{2 . 4}^{[18]}$ 设 $X$ 为集合且 $\mu_{A}: X \longrightarrow[0,1]$, $\nu_{A}: X \longrightarrow[0,1]$ 为两个映射, 若

$$
\mu_{A}(x)+\nu_{A}(x) \leqslant 1, \forall x \in X,
$$

则称 $A=\left(X, \mu_{A}, \nu_{A}\right)$ 为 $X$ 上的一个直觉模糊子集, 且记 $A(x)=\left(\mu_{A}(x), \nu_{A}(x)\right)$.
令 $I \mathcal{F}(X)$ 表示 $X$ 上的所有直觉模糊集的类, 在 $I \mathcal{F}(X)$ 中规定:

设 $A=\left(X, \mu_{A}, \nu_{A}\right), B=\left(X, \mu_{B}, \nu_{B}\right), A_{t}=(X$, $\left.\mu_{A_{t}}, \nu_{A_{t}}\right)$ 为集合 $X$ 上的直觉模糊集, 则有

(1) $A \subset B \Leftrightarrow \mu_{A}(x) \leqslant \mu_{B}(x), \nu_{A}(x) \geqslant \nu_{B}(x)$, $\forall x \in X$.

(2) $A^{c}=\left(X, \nu_{A}, \mu_{A}\right)$.

(3) $A \cup B=\left(X, \mu_{A \cup B}, \nu_{A \cup B}\right)$, 这里 $\mu_{A \cup B}(x)=$ $\mu_{A}(x) \vee \mu_{B}(x), \nu_{A \cup B}(x)=\nu_{A}(x) \wedge \nu_{B}(x)$.

(4) $A \cap B=\left(X, \mu_{A \cap B}, \nu_{A \cap B}\right)$, 这里 $\mu_{A \cap B}(x)=$ $\mu_{A}(x) \wedge \mu_{B}(x), \nu_{A \cap B}(x)=\nu_{A}(x) \vee \nu_{B}(x)$.

(5) 令 $A=\bigcup_{t \in T} A_{t}$, 则 $\mu_{A}(x)=\bigvee_{t \in T} \mu_{A_{t}}(x)$, $\nu_{A}(x)=\bigwedge_{t \in T} \nu_{A_{t}}(x)$.

(6) 令 $B=\bigcap_{t \in T} A_{t}$, 则 $\mu_{B}(x)=\bigwedge_{t \in T} \mu_{A_{t}}(x)$, $\nu_{B}(x)=\bigvee_{t \in T} \nu_{A_{t}}(x)$.

(7) 令 $\tilde{X}(x) \equiv(1,0), \forall x \in X ; \tilde{\emptyset}(x)=$ $(0,1), \forall x \in X$, 则 $(I \mathcal{F}(X), \cup, \cap, c, \tilde{X}, \tilde{\emptyset})$ 为一个 $F$ 格. 令 $L=\{(a, b) \mid a, b \in[0,1], a+b \leqslant 1\}$, 在 $L$ 中规 定:

(a) $\left(a_{1}, b_{1}\right) \leqslant\left(a_{2}, b_{2}\right) \Leftrightarrow a_{1} \leqslant a_{2}, b_{1} \geqslant b_{2}$.

(b) $\left(a_{1}, b_{1}\right) \vee\left(a_{2}, b_{2}\right)=\left(a_{1} \vee a_{2}, b_{1} \wedge b_{2}\right)$; $\left(a_{1}, b_{1}\right) \wedge\left(a_{2}, b_{2}\right)=\left(a_{1} \wedge a_{2}, b_{1} \vee b_{2}\right)$.

(c) $(a, b)^{c}=(b, a)$.

(d) $\bigvee_{t \in T}\left(a_{t}, b_{t}\right)=\left(\bigvee_{t \in T} a_{t}, \bigwedge_{t \in T} b_{t}\right)$; $\bigwedge_{t \in T}\left(a_{t}, b_{t}\right)=\left(\bigwedge_{t \in T} a_{t}, \bigvee_{t \in T} b_{t}\right)$.

(e) $\overline{1}=(1,0), \overline{0}=(0,1)$. 则 $(L, \vee, \wedge, c, \overline{1}, \overline{0})$ 为 $F$ 格. 于是直觉模糊集 $A=(X$, $\left.\mu_{A}, \nu_{A}\right)$ 可以看成一个 $L$ - 模糊集 ${ }^{[20,21]}$ :

$$
A: X \rightarrow L x \mapsto\left(\mu_{A}(x), \nu_{A}(x)\right) .
$$

为了方便, 本文也用 $L^{X}=\{A \mid A: X \rightarrow L$ 为映 射 $\}$ 来记 $I \mathcal{F}(X)$.

定义 $2.5^{[16,17]}$ 设 $X$ 为集合, $2^{X}$ 表示 $X$ 的幕 集. $H:[0,1] \rightarrow 2^{X}$ 为一个映射.

(1) 若 $\left(\lambda_{1}<\lambda_{2} \Rightarrow H\left(\lambda_{1}\right) \supset H\left(\lambda_{2}\right)\right)$, 则称 $H$ 为 $X$ 的反序集合套;

(2) 若 $\left(\lambda_{1}<\lambda_{2} \Rightarrow H\left(\lambda_{1}\right) \subset H\left(\lambda_{2}\right)\right)$, 则称 $H$ 为 $X$ 的顺序集合套.

定义 $\mathbf{2 . 6}^{[19]}$ 设 $X$ 为一个集合, 若 $A(x)=$ $\left[A^{-}(x), A^{+}(x)\right], \forall x \in X$, 则称 $A$ 为 $X$ 上的一个区 间值模糊集, 这里 $0 \leqslant A^{-}(x) \leqslant A^{+}(x) \leqslant 1$. 
令 $\bar{L}=\left\{\left[a^{-}, a^{+}\right] \mid 0 \leqslant a^{-} \leqslant a^{+} \leqslant 1\right\}, \bar{L}^{X}=$ $\{A \mid A: X \rightarrow \bar{L}$ 为映射 $\}$ 为区间值模糊集的类. 有关 区间值模糊集的运算请见文献 [26].

定义 $2.7^{[22]}$ 设 $A=\left(X, \mu_{A}, \nu_{A}\right)$ 为直觉模糊集, $\alpha, \beta \in[0,1], \alpha+\beta \leqslant 1$, 则称

$$
A_{[\alpha, \beta]}=\left\{x \mid \mu_{A}(x) \geqslant \alpha, \nu_{A}(x) \leqslant \beta\right\}
$$

为 $A$ 的 $[\alpha, \beta]$ - 截集.

定义 $2.8^{[23]}$ 设 $A \in \bar{L}^{X}$ 为 $X$ 上的区间 值模糊集, 且 $A(x)=\left[A^{-}(x), A^{+}(x)\right], \forall x \in X$. 设 $\lambda_{1}, \lambda_{2} \in[0,1], \lambda_{1} \leqslant \lambda_{2}$, 则

$$
\begin{aligned}
& A_{\left[\lambda_{1}, \lambda_{2}\right]}^{(1,1)}=\left\{x \mid x \in X, A^{-}(x) \geqslant \lambda_{1}, A^{+}(x) \geqslant \lambda_{2}\right\}, \\
& A_{\left[\lambda_{1}, \lambda_{2}\right]}^{(2,2)}=\left\{x \mid x \in X, A^{-}(x)>\lambda_{1}, A^{+}(x)>\lambda_{2}\right\},
\end{aligned}
$$

分别为 $A$ 的 $(1,1)$ - 截集和 $(2,2)$ - 截集.

有关这类区间值模糊集截集的性质和表现定理 请见文献 [23 25].

\section{3 直觉模糊集的截集}

我们先通过例子来说明: 文献 [23] 定义的区间 值模糊集截集的性质不理想.

例 3.1 设 $X=\{a, b\}, A(a)=[0.4,0.6], A(b)=$ $[0.4,0.7] ; B(a)=[0.4,0.7], B(b)=[0.5,0.6] ; C(a)=$ $[0.5,0.6], C(b)=[0.4,0.7] ; \alpha=[0.4,0.6], \beta=[0.3,0.7]$, $\left[\lambda_{1}, \lambda_{2}\right]=[0.4,0.6]$, 则 $A^{c}(a)=[0.4,0.6], A^{c}(b)=$ $[0.3,0.6], \alpha \wedge \beta=[0.3,0.6]$. 于是

$$
\left(A^{c}\right)_{\left[\lambda_{1}, \lambda_{2}\right]}^{(1,1)}=\{a\}, \quad\left(A_{\left[1-\lambda_{2}, 1-\lambda_{1}\right]}^{(2,2)}\right)^{c}=\{a, b\},
$$

即 $\left(A^{c}\right)_{\left[\lambda_{1}, \lambda_{2}\right]}^{(1,1)} \neq\left(A_{\left[1-\lambda_{2}, 1-\lambda_{1}\right]}^{(2,2)}\right)^{c}$. 因此这种截集不满 足文献 [16] 总结的模糊集截集的性质 6 .

由于 $(B \cup C)_{\left[\lambda_{1}, \lambda_{2}\right]}^{(2,2)}=\{a, b\}, B_{\left[\lambda_{1}, \lambda_{2}\right]}^{(2,2)}=\emptyset, C_{\left[\lambda_{1}, \lambda_{2}\right]}^{(2,2)}$ $=\emptyset$, 所以 $(B \cup C)_{\left[\lambda_{1}, \lambda_{2}\right]}^{(2,2)} \neq B_{\left[\lambda_{1}, \lambda_{2}\right]}^{(2,2)} \cup C_{\left[\lambda_{1}, \lambda_{2}\right]}^{(2,2)}$, 即 $(2,2)-$ 截集不保持 “并” 运算.

又 $A_{\alpha}^{(2,2)}=A_{\beta}^{(2,2)}=\emptyset, A_{\alpha \wedge \beta}^{(2,2)}=\{b\}$, 于是 $A_{\alpha}^{(2,2)}$ $\cup A_{\beta}^{(2,2)} \neq A_{\alpha \wedge \beta}^{(2,2)}$, 即文献 [16] 中的截集的性质 5 不成 立.

下面的例子说明文献 [24] 建立的表现定理不保 持 “余” 运算.

例 3.2 设 $X=\{a, b\}, A(a)=[0.4,0.6], A(b)=$
$[0.4,0.7] ; H\left(\alpha_{1}, \alpha_{2}\right)=A_{\left[\alpha_{1}, \alpha_{2}\right]}^{(1,1)}$, 则

$$
H\left(\alpha_{1}, \alpha_{2}\right)= \begin{cases}\{a, b\}, & \alpha_{1} \leqslant 0.4, \alpha_{2} \leqslant 0.6 ; \\ \{b\}, & \alpha_{1} \leqslant 0.4,0.6<\alpha_{2} \leqslant 0.7 ; \\ \emptyset, & \alpha_{2}>0.7 \text { 或者 } \\ & 0.4<\alpha_{1} \leqslant \alpha_{2} \leqslant 0.7 .\end{cases}
$$

按照文献 [24] 规定的集合套的运算规则有

$$
\begin{aligned}
& H^{c}\left(\alpha_{1}, \alpha_{2}\right)=\left(H\left(1-\alpha_{2}, 1-\alpha_{1}\right)\right)^{c} \\
& \quad= \begin{cases}\emptyset, & 0.4 \leqslant \alpha_{1}, 0.6 \leqslant \alpha_{2} ; \\
\{a\}, & 0.3 \leqslant \alpha_{1}<0.4,0.6 \leqslant \alpha_{2} ; \\
\{a, b\}, & \alpha_{1}<0.3 \text { 或者 }\end{cases} \\
& 0.3 \leqslant \alpha_{1} \leqslant \alpha_{2}<0.6 .
\end{aligned}
$$

按照文献 [24] 的表现定理有:

$$
\begin{aligned}
T\left(H^{c}\right)(b)= & \bigvee\left\{\left[\alpha_{1}, \alpha_{2}\right] \mid \alpha_{1}<0.3\right. \text { 或者 } \\
& \left.0.3 \leqslant \alpha_{1} \leqslant \alpha_{2}<0.6\right\} \\
= & {[0.6,1] . }
\end{aligned}
$$

由于 $T(H)(b)=A(b)=[0.4,0.7]$, 所以 $(T(H))^{c}(b)=$ $[0.3,0.6] \neq T\left(H^{c}\right)(b)$. 因此按照文献 $[23]$ 定义的截集 导出的表现定理不保持余运算.

文献 [22] 定义的截集也有类似的不足. 因此文 献 [22 25] 中的定义和结论没有完全推广模糊集截 集的相关定义和结论. 为此, 我们重新定义直觉模糊 集和区间值模糊集的截集. 先给出如下定义:

定义 3.1 设 $X$ 为一个集合, 称映射 $A: X \rightarrow$ $\left\{0, \frac{1}{2}, 1\right\}$ 为一个三值模糊集. $X$ 上的所有三值模糊 集的类记作 $3^{X}$.

在 $3^{X}$ 中采用 Zadeh 运算, 即: 对 $A, B, A_{t} \in 3^{X}$, $x \in X$, 令 $(A \cup B)(x)=A(x) \vee B(x) ;(A \cap B)(x)=$ $A(x) \wedge B(x) ; \quad A^{c}(x)=1-A(x) ; \quad\left(\bigcup_{t \in T} A_{t}\right)(x)=$ $\bigvee_{t \in T} A_{t}(x) ;\left(\bigcap_{t \in T} A_{t}\right)(x)=\bigwedge_{t \in T} A_{t}(x) ; X(x) \equiv$ $1, \forall x \in X ; \emptyset(x) \equiv 0, \forall x \in X$, 则 $\left(3^{X}, \cup, \cap, X, \emptyset\right)$ 为一个 $F$ 格.

利用三值模糊集, 可给出直觉模糊集的截集的定 义.

设 $A=\left(X, \mu_{A}, \nu_{A}\right)$ 为一个直觉模糊集且 $\lambda \in$ $[0,1]$, 对 $x \in X, \mu_{A}(x)$ 表示 $x$ 属于 $A$ 的程度, $\nu_{A}(x)$ 表示 $x$ 不属于 $A$ 的程度, 则 $1-\nu_{A}(x)$ 也 反映了 $x$ 属于 $A$ 的程度且 $\mu_{A}(x) \leqslant 1-\nu_{A}(x)$. 
于是 $\mu_{A}(x)$ 与 $1-\nu_{A}(x)$ 将 $[0,1]$ 分成了三部分: $\left[0, \mu_{A}(x)\right],\left(\mu_{A}(x), 1-\nu_{A}(x)\right],\left(1-\nu_{A}(x), 1\right]$. 为了表 现 $x$ 属于 $A$ 的程度与水平 $\lambda$ 的关系, 我们以水平 $\lambda$ 为阈值且这样考虑:

当 $\lambda \in\left[0, \mu_{A}(x)\right]$ 时, 相对水平 $\lambda$ 来说, $x$ 一定属 于 $A$. 因此 $x$ 属于 $A$ 的程度为 1 ;

当 $\lambda \in\left(1-\nu_{A}(x), 1\right]$ 时, 相对水平 $\lambda$ 来说, $x$ 一 定不属于 $A$, 因此 $x$ 属于 $A$ 的程度为 0 ;

当 $\lambda \in\left(\mu_{A}(x), 1-\nu_{A}(x)\right]$ 时, 相对水平 $\lambda$ 说, $x$ 既有属于 $A$ 的程度, 又有不属于 $A$ 的程度, 这时我 们认为 $x$ 属于 $A$ 的程度为 $\frac{1}{2}$.

在如此分析的基础上, 我们就可以给出直觉模糊

\section{集截集的定义:}

定义 3.2 若 $A_{\lambda}, A_{\underline{\lambda}} \in 3^{X}$ 且

$$
\begin{aligned}
& A_{\lambda}(x)= \begin{cases}1, & \mu_{A}(x) \geqslant \lambda ; \\
\frac{1}{2}, & \mu_{A}(x)<\lambda \leqslant 1-\nu_{A}(x) ; \\
0, & \lambda>1-\nu_{A}(x),\end{cases} \\
& A_{\underline{\lambda}}(x)= \begin{cases}1, & \mu_{A}(x)>\lambda ; \\
\frac{1}{2}, & \mu_{A}(x) \leqslant \lambda<1-\nu_{A}(x) ; \\
0, & \lambda \geqslant 1-\nu_{A}(x),\end{cases}
\end{aligned}
$$

则称 $A_{[\lambda]}$ 为 $A$ 的 $\lambda$-上截集, $A_{[\underline{ }}$ 为 $A$ 的 $\lambda$-强上截 集.

类似的有:

定义 3.3 若 $A^{\lambda}, A^{\lambda} \in 3^{X}$ 且

$$
\begin{aligned}
& A^{\lambda}(x)= \begin{cases}1, & \nu_{A}(x) \geqslant \lambda ; \\
\frac{1}{2}, & \nu_{A}(x)<\lambda \leqslant 1-\mu_{A}(x) ; \\
0, & \lambda>1-\mu_{A}(x),\end{cases} \\
& A^{\underline{\lambda}}(x)= \begin{cases}1, & \nu_{A}(x)>\lambda ; \\
\frac{1}{2}, & \nu_{A}(x) \leqslant \lambda<1-\mu_{A}(x) ; \\
0, & \lambda \geqslant 1-\mu_{A}(x),\end{cases}
\end{aligned}
$$

则称 $A^{\lambda}$ 为 $A$ 的 $\lambda$-下截集, $A^{\lambda}$ 为 $A$ 的 $\lambda$ - 强下截集.

定义 3.4 若 $A_{[\lambda]}, A_{[\underline{ }} \in 3^{X}$ 且

$$
A_{[\lambda]}(x)= \begin{cases}1, & \mu_{A}(x)+\lambda \geqslant 1 ; \\ \frac{1}{2}, & \nu_{A}(x) \leqslant \lambda<1-\mu_{A}(x) ; \\ 0, & \lambda<\nu_{A}(x),\end{cases}
$$

$$
A_{[\underline{\lambda}]}(x)= \begin{cases}1, & \mu_{A}(x)+\lambda>1 ; \\ \frac{1}{2}, & \nu_{A}(x)<\lambda \leqslant 1-\mu_{A}(x) ; \\ 0, & \lambda \leqslant \nu_{A}(x)\end{cases}
$$

则称 $A_{\lambda}$ 为 $A$ 的 $\lambda$-上重截集, $A_{\underline{\lambda}}$ 为 $A$ 的 $\lambda$-强上重 截集.

定义 3.5 若 $A^{[\lambda]}, A^{[\underline{ }} \underline{\underline{ }} \in 3^{X}$ 且

$$
\begin{aligned}
& A^{[\lambda]}(x)= \begin{cases}1, & \nu_{A}(x)+\lambda \geqslant 1 ; \\
\frac{1}{2}, & \mu_{A}(x) \leqslant \lambda<1-\nu_{A}(x) ; \\
0, & \lambda<\mu_{A}(x),\end{cases} \\
& A^{[\underline{ }]}(x)= \begin{cases}1, & \nu_{A}(x)+\lambda>1 ; \\
\frac{1}{2}, & \mu_{A}(x)<\lambda \leqslant 1-\nu_{A}(x) ; \\
0, & \lambda \leqslant \mu_{A}(x)\end{cases}
\end{aligned}
$$

则称 $A^{\lambda}$ 为 $A$ 的 $\lambda$-下重截集, $A^{\lambda}$ 为 $A$ 的 $\lambda$-强下重 截集.

注 3.1 (1) 定义 3.2 - 定义 3.5 给出的截集是 三值模糊集. 众所周知, 经典集合是以二值逻辑为 基础的. Zadeh 模糊集的截集是二值模糊集 (经典集 合). 因此, 从逻辑的观点看, 基于 Zadeh 模糊集的模 糊系统是以二值逻辑为基础的. 我们都知道, 在多值 逻辑中, 三值逻辑是一类非常重要的多值逻辑. 在 Lukasiewcz、Kleene 和 Gödel 三值逻辑系统中, 都可 将真值的赋值格视为 $\left\{0, \frac{1}{2}, 1\right\}^{[15]}$. 人们自然地会想 到：既然基于二值逻辑的集合为经典集 (二值集合), 那么基于三值逻辑的 “集合” 应为三值集合. 因此, 以 三值逻辑为基础的三值 (模糊) 集合应受到人们的重 视.

(2) 设 $\mathcal{F}(X)$ 为 Zadeh 模糊集的类, 则模糊集的 截集实际上是一个映射 ${ }^{[27]}$ :

$$
f: \mathcal{F}(X) \times[0,1] \rightarrow 2^{X} .
$$

(3) 定义 3.2 - 定义 3.5 说明: 直觉模糊集的截 集实际上是一个映射:

$$
F: I \mathcal{F}(X) \times[0,1] \rightarrow 3^{X} .
$$

(4) 当直觉模糊集 $A=\left(X, \mu_{A}, \nu_{A}\right)$ 满足: $\mu_{A}(x)+\nu_{A}(x)=1, \forall x \in X$ 时, 即 $A$ 退化为 Zadeh 模糊集时, 定义 3.2 - 定义 3.5 便与文献 [17] 中的四 类截集一致. 
因此, 我们给出的直觉模糊集的截集的定义是模 糊集截集定义的推广.

下面来讨论直觉模糊集截集的性质. 以下设 $A, B, A_{t}(t \in T)$ 均为集合 $X$ 上的直觉模糊集, $\lambda \in$ $[0,1]$. 则有:

性质 3.1 (1) $A_{\underline{\lambda}} \subset A_{\lambda}$.

(2) $\lambda_{1}<\lambda_{2} \Rightarrow A_{\lambda_{1}} \supset A_{\lambda_{2}}, A_{\underline{\lambda_{1}}} \supset A_{\underline{\lambda_{2}}}$, $A_{\underline{\lambda_{1}}} \supset A_{\lambda_{2}}$.

(3) $A \subset B \Rightarrow A_{\lambda} \subset B_{\lambda}, A_{\underline{\lambda}} \subset B_{\underline{\lambda}}$.

(4) $(A \cup B)_{\lambda}=A_{\lambda} \cup B_{\lambda},(A \cup B)_{\underline{\lambda}}=A_{\underline{\lambda}} \cup B_{\underline{\lambda}}$, $(A \cap B)_{\lambda}=A_{\lambda} \cap B_{\lambda},(A \cap B)_{\underline{\lambda}}=A_{\underline{\lambda}} \cap B_{\underline{\lambda}}$.

(5) $\left(A^{c}\right)_{\lambda}=\left(A_{\underline{1-\lambda}}\right)^{c},\left(A^{c}\right)_{\underline{\lambda}}=\left(A_{1-\lambda}\right)^{c}$.

(6) $\bigcup_{t \in T}\left(A_{t}\right)_{\lambda} \subset\left(\bigcup_{t \in T} A_{t}\right)_{\lambda}, \bigcup_{t \in T}\left(A_{t}\right)_{\underline{\lambda}}=$ $\left(\bigcup_{t \in T} A_{t}\right)_{\underline{\lambda}},\left(\bigcap_{t \in T} A_{t}\right)_{\lambda}=\bigcap_{t \in T}\left(A_{t}\right)_{\lambda},\left(\bigcap_{t \in T} A_{t}\right)_{\underline{\lambda}} \subset$ $\bigcap_{t \in T}\left(A_{t}\right)_{\underline{\lambda}}$.

(7) 令 $\lambda_{t} \in[0,1], a=\bigwedge_{t \in T} \lambda_{t}, b=\bigvee_{t \in T} \lambda_{t}$, 则 $\bigcup_{t \in T} A_{\lambda_{t}} \subset A_{a}, \bigcap_{t \in T} A_{\lambda_{t}}=A_{b}, \bigcup_{t \in T} A_{\underline{\lambda_{t}}}=A_{\underline{a}}$, $A_{\underline{b}} \subset \bigcap_{t \in T} A_{\underline{\lambda_{t}}}$.

(8) $A_{0}=X, A_{1}=\emptyset$.

证明 仅证 (5), 其他情况的证明是类似的.

(5) $\left(A^{c}\right)_{\lambda}(x)=1 \Leftrightarrow \nu_{A}(x) \geqslant \lambda=1-(1-\lambda) \Leftrightarrow$ $A_{\underline{1-\lambda}}(x)=0 \Leftrightarrow\left(A_{\underline{1-\lambda}}\right)^{c}(x)=1$.

$\left(A^{c}\right)_{\lambda}(x)=0 \Leftrightarrow \mu_{A}(x)>1-\lambda \Leftrightarrow \underline{A_{1-\lambda}}(x)=$ $1 \Leftrightarrow\left(\underline{A}_{\underline{1-\lambda}}\right)^{c}(x)=0$.

由于 $\left(A^{c}\right)_{\lambda},\left(A_{\underline{1-\lambda}}\right)^{c}$, 所以 $\left(A^{c}\right)_{\lambda}=\left(A_{\underline{1-\lambda}}\right)^{c}$. 同理可 证: $\left(A^{c}\right)_{\underline{\lambda}}=\left(A_{1-\lambda}\right)^{c}$.

性质 3.2 (1) $A^{\underline{\lambda}} \subset A^{\lambda}$.

(2) $\lambda_{1}<\lambda_{2} \Rightarrow A^{\lambda_{1}} \supset A^{\lambda_{2}}, A \underline{\lambda_{1}} \supset A$ 主2 $A \stackrel{\lambda_{1}}{\supset} A^{\lambda_{2}}$.

(3) $A \subset B \Rightarrow B^{\lambda} \subset A^{\lambda}, B^{\underline{\lambda}} \subset A^{\underline{\lambda}}$.

(4) $(A \cup B)^{\lambda}=A^{\lambda} \cap B^{\lambda},(A \cup B)^{\underline{\lambda}}=A^{\underline{\lambda}} \cap B^{\underline{\lambda}}$, $(A \cap B)^{\lambda}=A^{\lambda} \cup B^{\lambda},(A \cap B)^{\underline{\lambda}}=A^{\underline{\lambda}} \cup B^{\underline{\lambda}}$.

(5) $\left(A^{c}\right)^{\lambda}=\left(A^{1-\lambda}\right)^{c},\left(A^{c}\right)^{\underline{\lambda}}=\left(A^{1-\lambda}\right)^{c}$.

(6) $\bigcap_{t \in T}\left(A_{t}\right)^{\lambda}=\left(\bigcup_{t \in T} A_{t}\right)^{\lambda}, \bigcap_{t \in T}\left(A_{t}\right)^{\underline{\lambda}} \supset$ $\left(\bigcup_{t \in T} A_{t}\right)^{\underline{\lambda}},\left(\bigcap_{t \in T} A_{t}\right)^{\lambda} \supset \bigcup_{t \in T}\left(A_{t}\right)^{\lambda},\left(\bigcap_{t \in T} A_{t}\right)^{\underline{\lambda}}=$ $\bigcup_{t \in T}\left(A_{t}\right) \underline{\lambda}$.

(7) 令 $\lambda_{t} \in[0,1], a=\bigwedge_{t \in T} \lambda_{t}, b=\bigvee_{t \in T} \lambda_{t}$, 则 $\bigcup_{t \in T} A^{\lambda_{t}} \subset A^{a}, \bigcap_{t \in T} A^{\lambda_{t}}=A^{b}, \bigcup_{t \in T} A^{\lambda_{t}}=A^{\underline{a}}$, $A^{\underline{b}} \subset \bigcap_{t \in T} A \underline{\lambda_{t}}$.

(8) $A^{0}=X, A^{1}=\emptyset$.
性质 $3.3(1) A_{[\underline{ }} \subset A_{[\lambda]}$.

(2) $\lambda_{1}<\lambda_{2} \Rightarrow A_{\left[\lambda_{1}\right]} \subset A_{\left[\lambda_{2}\right]}, A_{\left[\underline{\left.\lambda_{1}\right]}\right.} \subset A_{\left[\underline{\left.\lambda_{2}\right]}\right.}$, $A_{\left[\lambda_{1}\right]} \subset A_{\left[\underline{\left.\lambda_{2}\right]}\right.}$.

(3) $A \subset B \Rightarrow A_{[\lambda]} \subset B_{[\lambda]}, A_{[\underline{\lambda]}} \subset B_{[\underline{[\lambda]}}$.

(4) $(A \cup B)_{[\lambda]}=A_{[\lambda]} \cup B_{[\lambda]},(A \cup B)_{[\underline{\lambda}]}=A_{[\underline{\lambda}]} \cup$ $B_{[\underline{\lambda}]},(A \cap B)_{[\lambda]}=A_{[\lambda]} \cap B_{[\lambda]},(A \cap B)_{[\underline{\lambda]}}=A_{[\underline{\lambda]}} \cap B_{[\underline{\lambda]}}$.

(5) $\left(A^{c}\right)_{[\lambda]}=\left(A_{[\underline{1-\lambda]}}\right)^{c},\left(A^{c}\right)_{[\underline{\lambda}]}=\left(A_{[1-\lambda]}\right)^{c}$.

(6) $\bigcup_{t \in T}\left(A_{t}\right)_{[\lambda]} \subset\left(\bigcup_{t \in T} A_{t}\right)_{[\lambda]}, \bigcup_{t \in T}\left(A_{t}\right)_{[\lambda]}=$ $\left(\bigcup_{t \in T} A_{t}\right)_{[\underline{\lambda}]},\left(\bigcap_{t \in T} A_{t}\right)_{[\lambda]}=\bigcap_{t \in T}\left(A_{t}\right)_{[\lambda]},\left(\bigcap_{t \in T} A_{t}\right)_{[\underline{\lambda}]}$ $\subset \bigcap_{t \in T}\left(A_{t}\right)_{[\underline{ }]}$.

(7) 令 $\lambda_{t} \in[0,1], a=\bigwedge_{t \in T} \lambda_{t}, b=\bigvee_{t \in T} \lambda_{t}$, 则 $\bigcup_{t \in T} A_{\left[\lambda_{t}\right]} \subset A_{[b]}, \bigcap_{t \in T} A_{\left[\lambda_{t}\right]}=A_{[a]}, \bigcup_{t \in T} A_{\left[\underline{\lambda_{t}}\right]}=$ $A_{[\underline{b}]}, A_{[\underline{a}]} \subset \bigcap_{t \in T} A_{\left[\underline{\lambda_{t}}\right]}$.

(8) $A_{[1]}=X, A_{[\underline{[0]}}=\emptyset$.

性质 $3.4(1) A^{[\underline{[\lambda]}} \subset A^{[\lambda]}$.

(2) $\lambda_{1}<\lambda_{2} \Rightarrow A^{\left[\lambda_{1}\right]} \subset A^{\left[\lambda_{2}\right]}, A^{\left[\underline{\lambda_{1}}\right]} \subset A^{\left[\underline{\lambda_{2}}\right]}$, $A^{\left[\lambda_{1}\right]} \subset A^{\left[\underline{\lambda_{2}}\right]}$.

(3) $A \subset B \Rightarrow B^{[\lambda]} \subset A^{[\lambda]}, B^{[\lambda]} \subset A^{[\underline{ }}$.

(4) $(A \cup B)^{[\lambda]}=A^{[\lambda]} \cap B^{[\lambda]},(A \cup B)^{[\lambda]}=A^{[\underline{\lambda}]} \cap$ $B^{[\underline{\underline{\lambda}}},(A \cap B)^{[\lambda]}=A^{[\lambda]} \cup B^{[\lambda]},(A \cap B)^{[\underline{\lambda}]}=A^{[\underline{\underline{\lambda}}]} \cup B^{[\underline{\underline{\lambda}}]}$.

(5) $\left(A^{c}\right)^{[\lambda]}=\left(A^{[\underline{1-\lambda}]}\right)^{c},\left(A^{c}\right)^{[\underline{\lambda}]}=\left(A^{[1-\lambda]}\right)^{c}$.

(6) $\bigcap_{t \in T}\left(A_{t}\right)^{[\lambda]}=\left(\bigcup_{t \in T} A_{t}\right)^{[\lambda]}, \bigcap_{t \in T}\left(A_{t}\right)^{[\underline{ }} \underline{\partial} \supset$ $\left(\bigcup_{t \in T} A_{t}\right)^{[\lambda]},\left(\bigcap_{t \in T} A_{t}\right)^{[\lambda]} \supset \bigcup_{t \in T}\left(A_{t}\right)^{[\lambda]},\left(\bigcap_{t \in T} A_{t}\right)^{[\underline{\lambda}]}$ $=\bigcup_{t \in T}\left(A_{t}\right)^{[\underline{\underline{\lambda}}]}$.

(7) 令 $\lambda_{t} \in[0,1], a=\bigwedge_{t \in T} \lambda_{t}, b=\bigvee_{t \in T} \lambda_{t}$, 则 $\bigcup_{t \in T} A^{\left[\lambda_{t}\right]} \subset A^{[b]}, \bigcap_{t \in T} A^{\left[\lambda_{t}\right]}=A^{[a]}, \bigcup_{t \in T} A^{\left[\lambda_{t}\right]}=$ $A^{[\underline{b}]}, A^{[\underline{a}]} \subset \bigcap_{t \in T} A^{\left[\underline{\lambda}_{t}\right]}$.

(8) $A^{[1]}=X, A^{[0]}=\emptyset$.

注 3.2 (1) 通过上述讨论可以看出, 每种截集都 有完全类似的性质.

(2) 由文献 [17] 知: 直觉模糊集的截集与 Zadeh 模糊集的截集有完全一样的性质.

\section{4 直觉模糊集的分解定理}

设 $A \in 3^{X}, \lambda \in[0,1]$, 我们先给出以下定义:

定义 4.1 设 $f_{i}:[0,1] \times 3^{X} \rightarrow L^{X},(\lambda, A) \mapsto$ 
$f_{i}(\lambda, A)$ 为映射 $(i=1,2, \ldots, 8)$, 其中

$$
\begin{aligned}
& f_{1}(\lambda, A)(x)= \begin{cases}(0,1), & A(x)=0 \\
(\lambda, 1-\lambda), & A(x)=1 \\
(0,1-\lambda), & A(x)=\frac{1}{2}\end{cases} \\
& f_{2}(\lambda, A)(x)= \begin{cases}(\lambda, 1-\lambda), & A(x)=0 \\
(1,0), & A(x)=1 \\
(\lambda, 0), & A(x)=\frac{1}{2} .\end{cases} \\
& f_{3}(\lambda, A)(x)= \begin{cases}(1-\lambda, \lambda), & A(x)=0 \\
(0,1), & A(x)=1 \\
(0, \lambda), & A(x)=\frac{1}{2}\end{cases} \\
& f_{4}(\lambda, A)(x)= \begin{cases}(1,0), & A(x)=0 \\
(1-\lambda, \lambda), & A(x)=1 \\
(1-\lambda, 0), & A(x)=\frac{1}{2} .\end{cases} \\
& f_{5}(\lambda, A)(x)= \begin{cases}(0,1), & A(x)=0 \\
(1-\lambda, \lambda), & A(x)=1 \\
(0, \lambda), & A(x)=\frac{1}{2}\end{cases} \\
& f_{6}(\lambda, A)(x)= \begin{cases}(1-\lambda, \lambda), & A(x)=0 \\
(1,0), & A(x)=1 \\
(1-\lambda, 0), & A(x)=\frac{1}{2} .\end{cases} \\
& f_{7}(\lambda, A)(x)= \begin{cases}(\lambda, 1-\lambda), & A(x)=0 \\
(0,1), & A(x)=1 \\
(0,1-\lambda), & A(x)=\frac{1}{2} .\end{cases} \\
& f_{8}(\lambda, A)(x)= \begin{cases}(1,0), & A(x)=0 \\
(\lambda, 1-\lambda), & A(x)=1 ; \\
(\lambda, 0), & A(x)=\frac{1}{2} .\end{cases}
\end{aligned}
$$

则我们有下列分解定理.

定理 4.1 设 $A=\left(X, \mu_{A}, \nu_{A}\right)$ 为直觉模糊集, 则

(1) $A=\bigcup_{\lambda \in[0,1]} f_{1}\left(\lambda, A_{\lambda}\right)=\bigcap_{\lambda \in[0,1]} f_{2}\left(\lambda, A_{\lambda}\right)$;

(2) $A=\bigcup_{\lambda \in[0,1]} f_{1}\left(\lambda, A_{\underline{\lambda}}\right)=\bigcap_{\lambda \in[0,1]} f_{2}\left(\lambda, A_{\underline{\lambda}}\right)$;
(3) 设映射 $H:[0,1] \rightarrow 3^{X}$ 满足: $A_{\underline{\lambda}} \subset H(\lambda) \subset$ $A_{\lambda}$, 则

(I) $A=\bigcup_{\lambda \in[0,1]} f_{1}(\lambda, H(\lambda))=\bigcap_{\lambda \in[0,1]} f_{2}(\lambda, H(\lambda))$;

(II) $\lambda_{1}<\lambda_{2} \Rightarrow H\left(\lambda_{1}\right) \supset H\left(\lambda_{2}\right)$;

(III) $A_{\lambda}=\bigcap_{\alpha<\lambda} H(\alpha), A_{\underline{\lambda}}=\bigcup_{\alpha>\lambda} H(\alpha)$.

证明 (1)

$\left(\bigcup_{\lambda \in[0,1]} f_{1}\left(\lambda, A_{\lambda}\right)\right)(x)=\bigvee_{\lambda \in[0,1]} f_{1}\left(\lambda, A_{\lambda}\right)(x)$

$=\left(\bigvee\left\{(\lambda, 1-\lambda) \mid \lambda \in[0,1]\right.\right.$ 且 $\left.\left.A_{\lambda}(x)=1\right\}\right)$

$\bigvee\left(\bigvee\left\{(0,1-\lambda) \mid \lambda \in[0,1]\right.\right.$ 且 $\left.\left.A_{\lambda}(x)=\frac{1}{2}\right\}\right)$

$=\left(\bigvee\left\{(\lambda, 1-\lambda) \mid \mu_{A}(x) \geqslant \lambda\right\}\right)$

$\bigvee\left(\bigvee\left\{(0,1-\lambda) \mid \mu_{A}(x)<\lambda \leqslant 1-\nu_{A}(x)\right\}\right)$

$=\left(\bigvee_{\mu_{A}(x) \geqslant \lambda} \lambda, \bigwedge_{\mu_{A}(x) \geqslant \lambda}(1-\lambda)\right)$

$\bigvee\left(0, \bigwedge\left\{1-\lambda \mid \mu_{A}(x)<\lambda \leqslant 1-\nu_{A}(x)\right\}\right)$

$=\left(\mu_{A}(x), 1-\mu_{A}(x)\right)$

$\bigvee\left(0, \wedge\left\{1-\lambda \mid 1-\mu_{A}(x)>1-\lambda \geqslant \nu_{A}(x)\right\}\right)$

$=\left(\mu_{A}(x), 1-\mu_{A}(x)\right) \bigvee\left(0, \nu_{A}(x)\right)$

$=\left(\mu_{A}(x), \nu_{A}(x)\right)=A(x)$.

因此 $A=\bigcup_{\lambda \in[0,1]} f_{1}\left(\lambda, A_{\lambda}\right)$.

类似的,

$\left(\bigcap_{\lambda \in[0,1]} f_{2}\left(\lambda, A_{\lambda}\right)\right)(x)=\bigwedge_{\lambda \in[0,1]} f_{2}\left(\lambda, A_{\lambda}\right)(x)$

$=\left(\bigwedge\left\{(\lambda, 1-\lambda) \mid \lambda \in[0,1]\right.\right.$ 且 $\left.\left.A_{\lambda}(x)=0\right\}\right)$

$\bigwedge\left(\bigwedge\left\{(\lambda, 0) \mid \lambda \in[0,1]\right.\right.$ 且 $\left.\left.A_{\lambda}(x)=\frac{1}{2}\right\}\right)$

$=\left(\bigwedge\left\{(\lambda, 1-\lambda) \mid \lambda>1-\nu_{A}(x)\right\}\right)$

$\bigwedge\left(\bigwedge\left\{\lambda \mid \mu_{A}(x)<\lambda \leqslant 1-\nu_{A}(x)\right\}, 0\right)$

$=\left(\bigwedge\left\{\lambda \mid \lambda>1-\nu_{A}(x)\right\}, \bigvee\left\{1-\lambda \mid \lambda>1-\nu_{A}(x)\right\}\right)$

$\bigwedge\left(\mu_{A}(x), 0\right)$

$=\left(1-\nu_{A}(x), \nu_{A}(x)\right) \bigwedge\left(\mu_{A}(x), 0\right)=\left(\mu_{A}(x), \nu_{A}(x)\right)$

$=A(x)$.

因此 $A=\bigcap_{\lambda \in[0,1]} f_{2}\left(\lambda, A_{\lambda}\right)$.

(2) 的证明与 (1) 类似.

(3) (I) 由于 $A_{\underline{\lambda}} \subset H(\lambda) \subset A_{\lambda}$, 所以 $f_{1}\left(\lambda, A_{\underline{\lambda}}\right) \subset$ $\left.f_{1}(\lambda, H(\lambda)) \subset f_{1}\left(\lambda, A_{\lambda}\right)\right), f_{2}\left(\lambda, A_{\underline{\lambda}}\right) \subset f_{2}(\lambda, H(\lambda)) \subset$ $\left.f_{2}\left(\lambda, A_{\lambda}\right)\right)$. 则

$$
\begin{aligned}
A= & \bigcup_{\lambda \in[0,1]} f_{1}\left(\lambda, A_{\underline{\lambda}}\right) \subset \bigcup_{\lambda \in[0,1]} f_{1}(\lambda, H(\lambda)) \\
& \subset \bigcup_{\lambda \in[0,1]} f_{1}\left(\lambda, A_{\lambda}\right)=A ; \\
A= & \bigcap_{\lambda \in[0,1]} f_{2}\left(\lambda, A_{\underline{\lambda}}\right) \subset \bigcap_{\lambda \in[0,1]} f_{2}(\lambda, H(\lambda)) \\
& \subset \bigcap_{\lambda \in[0,1]} f_{2}\left(\lambda, A_{\lambda}\right)=A .
\end{aligned}
$$

因此, $A=\bigcup_{\lambda \in[0,1]} f_{1}(\lambda, H(\lambda))=\bigcap_{\lambda \in[0,1]} f_{2}(\lambda$, $H(\lambda))$.

(II) $\lambda_{1}<\lambda_{2} \Rightarrow H\left(\lambda_{1}\right) \supset A_{\underline{\lambda_{1}}} \supset A_{\lambda_{2}} \supset H\left(\lambda_{2}\right)$. 
(III) 当 $\alpha<\lambda$ 时, 有 $H(\alpha) \supset A_{\underline{\alpha}} \supset A_{\lambda}$, 所以 $\bigcap_{\alpha<\lambda} H(\alpha) \supset A_{\lambda}$.

另一方面, 由 $H(\alpha) \subset A_{\alpha}$ 及性质 3.1(7) 知 $\bigcap_{\alpha<\lambda} H(\alpha) \subset \bigcap_{\alpha<\lambda} A_{\alpha}=A_{\lambda}$.

因此 $A_{\lambda}=\bigcap_{\alpha<\lambda} H(\alpha)$.

类似的, 当 $\alpha>\lambda$ 时, 有 $A_{\underline{\lambda}} \supset A_{\alpha} \supset H(\alpha)$, 所以 $A_{\underline{\lambda}} \supset \bigcup_{\alpha>\lambda} H(\alpha)$.

另一方面, 由 $H(\alpha) \supset A_{\underline{\alpha}}$ 及性质 3.1 (7) 知:

$$
\bigcup_{\alpha>\lambda} H(\alpha) \supset \bigcup_{\alpha>\lambda} A_{\underline{\alpha}}=A_{\underline{\lambda}} .
$$

因此 $A_{\underline{\lambda}}=\bigcup_{\alpha>\lambda} H(\alpha)$.

定理 4.2 设 $A=\left(X, \mu_{A}, \nu_{A}\right)$ 为直觉模糊集, 则

(1) $A=\bigcup_{\lambda \in[0,1]} f_{3}\left(\lambda, A^{\lambda}\right)=\bigcap_{\lambda \in[0,1]} f_{4}\left(\lambda, A^{\lambda}\right)$;

(2) $A=\bigcup_{\lambda \in[0,1]} f_{3}\left(\lambda, A^{\underline{\lambda}}\right)=\bigcap_{\lambda \in[0,1]} f_{4}\left(\lambda, A^{\lambda}\right)$;

(3) 设映射 $H:[0,1] \rightarrow 3^{X}$ 满足: $A^{\underline{\lambda}} \subset H(\lambda) \subset$ $A^{\lambda}$, 则

(I) $A=\bigcup_{\lambda \in[0,1]} f_{3}(\lambda, H(\lambda))=\bigcap_{\lambda \in[0,1]} f_{4}(\lambda, H(\lambda))$;

(II) $\lambda_{1}<\lambda_{2} \Rightarrow H\left(\lambda_{1}\right) \supset H\left(\lambda_{2}\right)$;

(III) $A^{\lambda}=\bigcap_{\alpha<\lambda} H(\alpha), A^{\lambda}=\bigcup_{\alpha>\lambda} H(\alpha)$.

证明 (1)

$\left(\bigcup_{\lambda \in[0,1]} f_{3}\left(\lambda, A^{\lambda}\right)\right)(x)=\bigvee_{\lambda \in[0,1]} f_{3}\left(\lambda, A^{\lambda}\right)(x)$

$=\left(\bigvee\left\{(1-\lambda, \lambda) \mid \lambda \in[0,1]\right.\right.$ 且 $\left.\left.A^{\lambda}(x)=0\right\}\right)$

$\bigvee\left(\bigvee\left\{(0, \lambda) \mid \lambda \in[0,1]\right.\right.$ 且 $\left.\left.A^{\lambda}(x)=\frac{1}{2}\right\}\right)$

$=\left(\bigvee\left\{(1-\lambda, \lambda) \mid \lambda \in[0,1]\right.\right.$ 且 $\left.\left.\lambda>1-\mu_{A}(x)\right\}\right)$

$\bigvee\left(\bigvee\left\{(0, \lambda) \mid \nu_{A}(x)<\lambda \leqslant 1-\mu_{A}(x)\right\}\right)$

$=\left(\bigvee_{\lambda>1-\mu_{A}(x)}(1-\lambda), \bigwedge_{\lambda>1-\mu_{A}(x)} \lambda\right)$

$\bigvee\left(0, \bigwedge\left\{\lambda \mid \nu_{A}(x)<\lambda \leqslant 1-\mu_{A}(x)\right\}\right)$

$=\left(\mu_{A}(x), 1-\mu_{A}(x)\right) \bigvee\left(0, \nu_{A}(x)\right)=\left(\mu_{A}(x), \nu_{A}(x)\right)$

$=A(x)$.

因此 $A=\bigcup_{\lambda \in[0,1]} f_{3}\left(\lambda, A^{\lambda}\right)$. 类似的,

$\left(\bigcap_{\lambda \in[0,1]} f_{4}\left(\lambda, A^{\lambda}\right)\right)(x)=\bigwedge_{\lambda \in[0,1]} f_{4}\left(\lambda, A^{\lambda}\right)(x)$

$=\left(\bigwedge\left\{(1-\lambda, \lambda) \mid \lambda \in[0,1]\right.\right.$ 且 $\left.\left.A^{\lambda}(x)=1\right\}\right)$

$\bigwedge\left(\bigwedge\left\{(1-\lambda, 0) \mid \lambda \in[0,1]\right.\right.$ 且 $\left.\left.A^{\lambda}(x)=\frac{1}{2}\right\}\right)$

$=\left(\bigwedge\left\{(1-\lambda, \lambda) \mid \nu_{A}(x) \geqslant \lambda\right\}\right)$

$\bigwedge\left(\bigwedge\left\{(1-\lambda, 0) \mid \nu_{A}(x)<\lambda \leqslant 1-\mu_{A}(x)\right\}\right.$

$=\left(\bigwedge_{\nu_{A}(x) \geqslant \lambda}(1-\lambda), \bigvee_{\nu_{A}(x) \geqslant \lambda} \lambda\right)$

$\bigwedge\left(\bigwedge\left\{1-\lambda \mid \nu_{A}(x)<\lambda \leqslant 1-\mu_{A}(x)\right\}, 0\right)$

$=\left(1-\nu_{A}(x), \nu_{A}(x)\right) \bigwedge\left(\mu_{A}(x), 0\right)=\left(\mu_{A}(x), \nu_{A}(x)\right)$

$=A(x)$.
因此 $A=\bigcap_{\lambda \in[0,1]} f_{4}\left(\lambda, A^{\lambda}\right)$.

(2) 的证明与 (1) 类似.

(3) (I) 由于 $A^{\underline{\lambda}} \subset H(\lambda) \subset A^{\lambda}$, 所以 $f_{3}\left(\lambda, A^{\underline{\lambda}}\right) \supset$ $f_{3}(\lambda, H(\lambda)) \supset f_{3}\left(\lambda, A^{\lambda}\right), f_{4}\left(\lambda, A^{\underline{\lambda}}\right) \supset f_{4}(\lambda, H(\lambda)) \supset$ $f_{4}\left(\lambda, A^{\lambda}\right)$. 于是

$$
\begin{aligned}
A= & \bigcup_{\lambda \in[0,1]} f_{3}\left(\lambda, A^{\underline{\lambda}}\right) \supset \bigcup_{\lambda \in[0,1]} f_{3}(\lambda, H(\lambda)) \\
& \supset \bigcup_{\lambda \in[0,1]} f_{3}\left(\lambda, A^{\lambda}\right)=A ; \\
A= & \bigcap_{\lambda \in[0,1]} f_{4}\left(\lambda, A^{\lambda}\right) \supset \bigcap_{\lambda \in[0,1]} f_{4}(\lambda, H(\lambda)) \\
& \supset \bigcap_{\lambda \in[0,1]} f_{4}\left(\lambda, A^{\lambda}\right)=A .
\end{aligned}
$$

因此, $A=\bigcup_{\lambda \in[0,1]} f_{3}(\lambda, H(\lambda))=\bigcap_{\lambda \in[0,1]} f_{4}(\lambda$, $H(\lambda))$.

(II) $\lambda_{1}<\lambda_{2} \Rightarrow H\left(\lambda_{1}\right) \supset A \underline{\lambda_{1}} \supset A^{\lambda_{2}} \supset H\left(\lambda_{2}\right)$.

(III) 当 $\alpha<\lambda$ 时, 有 $H(\alpha) \supset A^{\underline{\alpha}} \supset A^{\lambda}$, 所以 $\bigcap_{\alpha<\lambda} H(\alpha) \supset A^{\lambda}$.

另一方面, 由 $H(\alpha) \subset A^{\alpha}$ 及性质 $3.2(7)$ 知

$$
\bigcap_{\alpha<\lambda} H(\alpha) \subset \bigcap_{\alpha<\lambda} A^{\alpha}=A^{\lambda} .
$$

因此 $A^{\lambda}=\bigcap_{\alpha<\lambda} H(\alpha)$.

类似的, 当 $\alpha>\lambda$ 时, 有 $A^{\lambda} \supset A^{\alpha} \supset H(\alpha)$, 所以 $A^{\underline{\lambda}} \supset \bigcup_{\alpha>\lambda} H(\alpha)$.

另一方面, 由 $H(\alpha) \supset A^{\underline{\alpha}}$ 及性质 $3.2(7)$ 知

$$
\bigcup_{\alpha>\lambda} H(\alpha) \supset \bigcup_{\alpha>\lambda} A^{\underline{\alpha}}=A^{\underline{\lambda}} \text {. }
$$

因此 $A^{\underline{\lambda}}=\bigcup_{\alpha>\lambda} H(\alpha)$.

定理 4.3 设 $A=\left(X, \mu_{A}, \nu_{A}\right)$ 为直觉模糊集, 则

(1) $A=\bigcup_{\lambda \in[0,1]} f_{5}\left(\lambda, A_{[\lambda]}\right)=\bigcap_{\lambda \in[0,1]} f_{6}\left(\lambda, A_{[\lambda]}\right)$;

(2) $A=\bigcup_{\lambda \in[0,1]} f_{5}\left(\lambda, A_{[\underline{\lambda}]}\right)=\bigcap_{\lambda \in[0,1]} f_{6}\left(\lambda, A_{[\underline{\lambda}]}\right)$;

(3) 设映射 $H:[0,1] \rightarrow 3^{X}$ 满足: $A_{[\lambda]} \subset H(\lambda) \subset$ $A_{[\lambda]}$, 则

(I) $A=\bigcup_{\lambda \in[0,1]} f_{5}(\lambda, H(\lambda))=\bigcap_{\lambda \in[0,1]} f_{6}(\lambda, H(\lambda))$;

(II) $\lambda_{1}<\lambda_{2} \Rightarrow H\left(\lambda_{1}\right) \subset H\left(\lambda_{2}\right)$;

(III) $A_{[\lambda]}=\bigcap_{\alpha>\lambda} H(\alpha), \quad A_{[\underline{\lambda}]}=\bigcup_{\alpha<\lambda} H(\alpha)$.

证明 (1)

$\left(\bigcup_{\lambda \in[0,1]} f_{5}\left(\lambda, A_{[\lambda]}\right)\right)(x)$

$=\bigvee_{\lambda \in[0,1]} f_{5}\left(\lambda, A_{[\lambda]}\right)(x)$

$=\left(\bigvee\left\{(1-\lambda, \lambda) \mid \lambda \in[0,1]\right.\right.$ 且 $\left.\left.A_{[\lambda]}(x)=1\right\}\right)$

$\bigvee\left(\bigvee\left\{(0, \lambda) \mid \lambda \in[0,1]\right.\right.$ 且 $\left.\left.A_{[\lambda]}(x)=\frac{1}{2}\right\}\right)$

$=\left(\bigvee\left\{(1-\lambda, \lambda) \mid \mu_{A}(x) \geqslant 1-\lambda\right\}\right)$

$\bigvee\left(\bigvee\left\{(0, \lambda) \mid \nu_{A}(x) \leqslant \lambda<1-\mu_{A}(x)\right\}\right)$ 


$$
\begin{aligned}
= & \left(\bigvee_{\lambda+\mu_{A}(x) \geqslant 1}(1-\lambda), \bigwedge_{\lambda+\mu_{A}(x) \geqslant 1} \lambda\right) \\
& \bigvee\left(0, \bigwedge\left\{\lambda \mid \nu_{A}(x) \leqslant \lambda<1-\mu_{A}(x)\right\}\right) \\
= & \left(\mu_{A}(x), 1-\mu_{A}(x)\right) \bigvee\left(0, \nu_{A}(x)\right)=\left(\mu_{A}(x), \nu_{A}(x)\right) \\
= & A(x) .
\end{aligned}
$$$$
\text { 因此 } A=\bigcup_{\lambda \in[0,1]} f_{5}\left(\lambda, A_{[\lambda]}\right) \text {. }
$$

类似的,

$\left(\bigcap_{\lambda \in[0,1]} f_{6}\left(\lambda, A_{[\lambda]}\right)\right)(x)=\bigwedge_{\lambda \in[0,1]} f_{6}\left(\lambda, A_{[\lambda]}\right)(x)$

$=\left(\bigwedge\left\{(1-\lambda, \lambda) \mid \lambda \in[0,1]\right.\right.$ 且 $\left.\left.A_{[\lambda]}(x)=0\right\}\right)$

$$
\bigwedge\left(\bigwedge\left\{(1-\lambda, 0) \mid \lambda \in[0,1] \text { 且 } A_{[\lambda]}(x)=\frac{1}{2}\right\}\right)
$$

$=\left(\bigwedge\left\{(1-\lambda, \lambda) \mid \nu_{A}(x)>\lambda\right\}\right)$

$$
\bigwedge\left(\bigwedge\left\{(1-\lambda, 0) \mid \nu_{A}(x)<\lambda \leqslant 1-\mu_{A}(x)\right\}\right)
$$

$=\left(\bigwedge_{\nu_{A}(x)>\lambda}(1-\lambda), \bigvee_{\nu_{A}(x)>\lambda} \lambda\right)$

$\bigwedge\left(\bigwedge\left\{1-\lambda \mid \nu_{A}(x)<\lambda \leqslant 1-\mu_{A}(x)\right\}, 0\right)$

$=\left(1-\nu_{A}(x), \nu_{A}(x)\right) \bigwedge\left(\mu_{A}(x), 0\right)=\left(\mu_{A}(x), \nu_{A}(x)\right)$

$=A(x)$.

因此 $A=\bigcap_{\lambda \in[0,1]} f_{6}\left(\lambda, A_{[\lambda]}\right)$.

其余部分的证明是显然的.

定理 4.4 设 $A=\left(X, \mu_{A}, \nu_{A}\right)$ 为直觉模糊集, 则

(1) $A=\bigcup_{\lambda \in[0,1]} f_{7}\left(\lambda, A^{[\lambda]}\right)=\bigcap_{\lambda \in[0,1]} f_{8}\left(\lambda, A^{[\lambda]}\right)$;

(2) $\left.A=\bigcup_{\lambda \in[0,1]} f_{7}\left(\lambda, A^{[\underline{\lambda}]}\right)=\bigcap_{\lambda \in[0,1]} f_{8}\left(\lambda, A^{[\underline{ }} \underline{ }\right]\right)$;

(3) 设映射 $H:[0,1] \rightarrow 3^{X}$ 满足: $A^{[\lambda]} \subset H(\lambda) \subset$ $A^{[\lambda]}$, 则

$$
\text { (I) } \begin{aligned}
A & =\bigcup_{\lambda \in[0,1]} f_{7}(\lambda, H(\lambda)) \\
& =\bigcap_{\lambda \in[0,1]} f_{8}(\lambda, H(\lambda)) ;
\end{aligned}
$$

(II) $\lambda_{1}<\lambda_{2} \Rightarrow H\left(\lambda_{1}\right) \subset H\left(\lambda_{2}\right)$;

(III) $A^{[\lambda]}=\bigcap_{\alpha>\lambda} H(\alpha), \quad A^{[\lambda]}=\bigcup_{\alpha<\lambda} H(\alpha)$.

证明 (1) $\left(\bigcup_{\lambda \in[0,1]} f_{7}\left(\lambda, A^{[\lambda]}\right)\right)(x)$

$=\bigvee_{\lambda \in[0,1]} f_{7}\left(\lambda, A^{[\lambda]}\right)(x)$

$=\left(\bigvee\left\{(\lambda, 1-\lambda) \mid \lambda \in[0,1]\right.\right.$ 且 $\left.\left.A^{[\lambda]}(x)=0\right\}\right)$

$\bigvee\left(\bigvee\left\{(0,1-\lambda) \mid \lambda \in[0,1]\right.\right.$ 且 $\left.\left.A^{[\lambda]}(x)=\frac{1}{2}\right\}\right)$

$=\left(\bigvee\left\{(\lambda, 1-\lambda) \mid \mu_{A}(x)>\lambda\right)\right.$

$\bigvee\left(\bigvee\left\{(0,1-\lambda) \mid \mu_{A}(x) \leqslant \lambda<1-\nu_{A}(x)\right\}\right)$

$=\left(\bigvee_{\mu_{A}(x)>\lambda} \lambda, \bigwedge_{\mu_{A}(x)>\lambda}(1-\lambda)\right)$

$\bigvee\left(0, \bigwedge\left\{1-\lambda \mid \mu_{A}(x) \leqslant \lambda<1-\nu_{A}(x)\right\}\right)$

$=\left(\mu_{A}(x), 1-\mu_{A}(x)\right) \bigvee\left(0, \nu_{A}(x)\right)=\left(\mu_{A}(x), \nu_{A}(x)\right)$

$=A(x)$.

因此 $A=\bigcup_{\lambda \in[0,1]} f_{7}\left(\lambda, A^{[\lambda]}\right)$.

类似的,

$\left(\bigcap_{\lambda \in[0,1]} f_{8}\left(\lambda, A^{[\lambda]}\right)\right)(x)=\bigwedge_{\lambda \in[0,1]} f_{8}\left(\lambda, A^{[\lambda]}\right)(x)$

$=\left(\bigwedge\left\{(\lambda, 1-\lambda) \mid A^{[\lambda]}(x)=1\right\}\right)$

$$
\begin{aligned}
& \bigwedge\left(\bigwedge\left\{(\lambda, 0) \mid A^{[\lambda]}(x)=\frac{1}{2}\right\}\right) \\
= & \left(\bigwedge\left\{(\lambda, 1-\lambda) \mid \nu_{A}(x) \geqslant 1-\lambda\right\}\right) \\
& \bigwedge\left(\bigwedge\left\{(\lambda, 0) \mid \mu_{A}(x) \leqslant \lambda<1-\nu_{A}(x)\right\}\right. \\
= & \left(\bigwedge_{\nu_{A}(x) \geqslant 1-\lambda} \lambda, \bigvee_{\nu_{A}(x) \geqslant 1-\lambda}(1-\lambda)\right) \\
& \bigwedge\left(\bigwedge\left\{\lambda \mid \mu_{A}(x) \leqslant \lambda<1-\nu_{A}(x)\right\}, 0\right) \\
= & \left(1-\nu_{A}(x), \nu_{A}(x)\right) \bigwedge\left(\mu_{A}(x), 0\right)=\left(\mu_{A}(x), \nu_{A}(x)\right) \\
= & A(x) .
\end{aligned}
$$

因此 $A=\bigcap_{\lambda \in[0,1]} f_{8}\left(\lambda, A^{[\lambda]}\right)$.

其余部分的证明是显然的.

注 4.1 (1) 从定理 4.1一定理 4.4 知: 我们已 经建立了直觉模糊集的分解定理.

(2) 在定义 4.1 中, 如果我们用 $\lambda A$ 和 $\lambda \circ A$ 分别 记 $f_{1}(\lambda, A)$ 和 $f_{2}(\lambda, A)$, 即:

$$
\lambda A \triangleq f_{1}(\lambda, A), \lambda \circ A \triangleq f_{2}(\lambda, A) .
$$

则定理 4.1 可以写成:

及

$$
A=\bigcup_{\lambda \in[0,1]} \lambda A_{\lambda}=\bigcap_{\lambda \in[0,1]} \lambda \circ A_{\lambda}
$$

$$
A=\bigcup_{\lambda \in[0,1]} \lambda H(\lambda)=\bigcap_{\lambda \in[0,1]} \lambda \circ H(\lambda) .
$$

这与模糊集的分解定理的表达式完全一致 ${ }^{[16,17]}$.

(3) 对定理 4.2- 定理 4.4 也可以同样进行表示. 因此我们得出以下结论: 对于直觉模糊集来说, 每种 截集都对应两种分解定理, 因此直觉模糊集共有八种 分解定理.

\section{5 直觉模糊集的表现定理}

为了建立直觉模糊集的表现定理, 我们先给出集 合套的定义:

定义 5.1 设 $H:[0,1] \rightarrow 3^{X}$ 为一映射, 若:

$$
\lambda_{1}<\lambda_{2} \Rightarrow H\left(\lambda_{1}\right) \supset H\left(\lambda_{2}\right),
$$

则称 $H$ 为 $X$ 上的一个三值反序集合套, $X$ 上的所 有三值反序集合套的类记作 $\mathcal{U}(X)$.

在 $\mathcal{U}(X)$ 中规定:

(a) $H_{1} \subset H_{2} \Leftrightarrow H_{1}(\alpha) \subset H_{2}(\alpha), \forall \alpha \in[0,1]$.

(b) $\bigcup_{t \in T} H_{t}:\left(\bigcup_{t \in T} H_{t}\right)(\alpha)=\bigcup_{t \in T} H_{t}(\alpha)$; $\bigcap_{t \in T} H_{t}:\left(\bigcap_{t \in T} H_{t}\right)(\alpha)=\bigcap_{t \in T} H_{t}(\alpha)$.

(c) $H^{c}: H^{c}(\alpha)=(H(1-\alpha))^{c} ; \bar{X}(\alpha) \equiv X, \bar{\emptyset}(\alpha) \equiv$ $\emptyset, \forall \alpha \in[0,1]$.

则 $(\mathcal{U}(X), \cup, \cap, c, \bar{X}, \bar{\emptyset})$ 为一个 $F$ 格. 
于是有下面的定理:

定理 5.1 设 $T_{i}: \mathcal{U}(X) \rightarrow L^{X}$ 为映射 $(i=1,2)$, 其中 $T_{1}(H)=\bigcup_{\lambda \in[0,1]} f_{1}(\lambda, H(\lambda)), T_{2}(H)=$ $\bigcap_{\lambda \in[0,1]} f_{2}(\lambda, H(\lambda))$. 则

(1) $T_{1}(H)=T_{2}(H)$;

(2) $T_{1}(H)_{\lambda}=\bigcap_{\alpha<\lambda} H(\alpha), T_{1}(H)_{\underline{\lambda}}$ $=\bigcup_{\alpha>\lambda} H(\alpha)$.

(3) $T_{1}\left(T_{2}\right)$ 为满射且 $T_{1}\left(\bigcup_{t \in T} H_{t}\right)=\bigcup_{t \in T} T_{1}\left(H_{t}\right)$; $T_{1}\left(\bigcap_{t \in T} H_{t}\right)=\bigcap_{t \in T} T_{1}\left(H_{t}\right) ; T_{1}\left(H^{c}\right)=\left(T_{1}(H)\right)^{c}$.

证明 (1) 设 $A=T_{1}(H)$. 我们先证 $A_{\underline{\lambda}} \subset$ $H(\lambda) \subset A_{\lambda}$. 事实上, 由于 $T_{1}(H)(x)=(\bigvee\{(\alpha, 1-$ $\alpha) \mid \alpha \in[0,1]$ 且 $H(\alpha)(x)=1\}) \bigvee(\bigvee\{(0,1-\alpha) \mid \alpha \in$ $[0,1]$ 且 $\left.\left.H(\alpha)(x)=\frac{1}{2}\right\}\right)=(\bigvee\{\alpha \mid \alpha \in[0,1]$ 且 $H(\alpha)(x)=1\}, \bigwedge\left\{1-\alpha \mid \alpha \in[0,1]\right.$ 且 $\left.\left.H(\alpha)(x) \geqslant \frac{1}{2}\right\}\right)$. 因此 $\mu_{A}(x)=\bigvee\{\alpha \mid \alpha \in[0,1]$ 且 $H(\alpha)(x)=1\}$, $\nu_{A}(x)=\bigwedge\left\{1-\alpha \mid \alpha \in[0,1]\right.$ 且 $\left.H(\alpha)(x) \geqslant \frac{1}{2}\right\}$.

当 $H(\lambda)(x)=1$ 时, $\mu_{A}(x)=\bigvee\{\alpha \mid \alpha \in[0,1]$ 且 $H(\alpha)(x)=1\} \geqslant \lambda$;

当 $A_{\lambda}(x)=0$ 时, $\nu_{A}(x)=\bigwedge\{1-\alpha \mid \alpha \in[0,1]$ 且 $\left.H(\alpha)(x) \geqslant \frac{1}{2}\right\}>1-\lambda$, 则 $H(\lambda)(x)=0$.

由于 $A_{\lambda}, H(\lambda) \in 3^{X}$, 所以 $H(\lambda) \subset A_{\lambda}$.

另一方面, 当 $A_{\underline{\lambda}}(x)=1$ 时, 有 $\mu_{A}(x)=\bigvee\{\alpha \mid \alpha \in$ $[0,1]$ 且 $H(\alpha)(x)=1\}>\lambda$, 则存在 $\alpha>\lambda$ 使 $H(\alpha)(x)=1$, 于是 $H(\lambda)(x) \geqslant H(\alpha)(x)=1$, 即 $H(\lambda)(x)=1$.

当 $H(\lambda)(x)=0$ 时, 只要 $H(\alpha)(x) \geqslant \frac{1}{2}$ 就一 定有 $\alpha<\lambda$. 于是 $\nu_{A}(x)=\bigwedge\{1-\alpha \mid \alpha \in[0,1]$ 且 $\left.H(\alpha)(x) \geqslant \frac{1}{2}\right\} \geqslant \bigwedge\{1-\alpha \mid \alpha<\lambda\}=1-\lambda$. 则 $A_{\underline{\lambda}}(x)=0$.

由于 $A_{\lambda}, H(\lambda) \in 3^{X}$, 所以 $A_{\underline{\lambda}} \subset H(\lambda)$.

因此 $A_{\underline{\lambda}} \subset H(\lambda) \subset A_{\lambda}$.

由定理 4.1 知: $T_{1}(H)=T_{2}(H)$.

(2) 由定理 4.1 知: $T_{1}(H)_{\lambda}=\bigcap_{\alpha<\lambda} H(\alpha)$, $T_{1}(H)_{\underline{\lambda}}=\bigcup_{\alpha>\lambda} H(\alpha)$.

(3) 设 $A \in L^{X}$, 令 $H \in \mathcal{U}(X)$ 且 $H(\lambda)=A_{\lambda}$. 由 定理 4.1 知: $T_{1}(H)=A$. 因此 $T_{1}$ 为满射. 下面证明 $T_{1}$ 保持运算. 设 $H_{t} \in \mathcal{U}(X)(t \in T)$, 则由性质 3.1 有 $T_{1}\left(\bigcup_{t \in T} H_{t}\right)_{\underline{\lambda}}=\bigcup_{\alpha>\lambda}\left(\bigcup_{t \in T} H_{t}\right)(\alpha)$

$=\bigcup_{\alpha>\lambda} \bigcup_{t \in T} H_{t}(\alpha)=\bigcup_{t \in T} \bigcup_{\alpha>\lambda} H_{t}(\alpha)$

$=\bigcup_{t \in T} T_{1}\left(H_{t}\right)_{\underline{\lambda}}=\left(\bigcup_{t \in T} T_{1}\left(H_{t}\right)\right)_{\underline{\lambda}}$.

$$
\begin{aligned}
T_{1} & \left(\bigcap_{t \in T} H_{t}\right)_{\lambda}=\bigcap_{\alpha<\lambda}\left(\bigcap_{t \in T} H_{t}\right)(\alpha) \\
& =\bigcap_{\alpha<\lambda} \bigcap_{t \in T} H_{t}(\alpha)=\bigcap_{t \in T} \bigcap_{\alpha<\lambda} H_{t}(\alpha) \\
& =\bigcap_{t \in c T} T_{1}\left(H_{t}\right)_{\lambda}=\left(\bigcap_{t \in T} T_{1}\left(H_{t}\right)\right)_{\lambda} .
\end{aligned}
$$

由定理 4.1 知:

$$
\begin{aligned}
& T_{1}\left(\bigcup_{t \in T} H_{t}\right)=\bigcup_{t \in T} T_{1}\left(H_{t}\right), T_{1}\left(\bigcap_{t \in T} H_{t}\right) \\
& =\bigcap_{t \in T} T_{1}\left(H_{t}\right) . \\
& \quad \text { 类似的, 有 }
\end{aligned}
$$

$$
\begin{aligned}
& T_{1}\left(H^{c}\right)_{\lambda}=\bigcap_{\alpha<\lambda} H^{c}(\alpha)=\bigcap_{\alpha<\lambda}(H(1-\alpha))^{c} \\
& \quad=\bigcup_{1-\alpha>1-\lambda}(H(1-\alpha))^{c}=\left(T_{1}(H)_{\underline{1-\lambda}}\right)^{c} \\
& \quad=\left(\left(T_{1}(H)\right)^{c}\right)_{\lambda} .
\end{aligned}
$$

由定理 4.1 (1) 知: $T_{1}\left(H^{c}\right)=\left(T_{1}(H)\right)^{c}$.

由以上讨论知: $T_{1}$ 保持运算.

推论 5.1 在 $\mathcal{U}(X)$ 中定义关系

$$
H_{1} \sim H_{2} \Leftrightarrow T_{1}\left(H_{1}\right)=T_{1}\left(H_{2}\right),
$$

则 $\sim$ 为 $\mathcal{U}(X)$ 上一个等价关系, 且商集 $\mathcal{U}(X) / \sim \cong$ $L^{X}$. 类.

因此，直觉模糊集实际上是三值集合套的等价

定理 5.1 是在定理 4.1 的基础上建立起来的. 如 果以定理 4.2 为基础可得下列定理:

定理 5.2 设 $T_{i}: \mathcal{U}(X) \rightarrow L^{X}$ 为映射 $(i=3,4)$, 其中

$T_{3}(H)=\bigcup_{\lambda \in[0,1]} f_{3}(\lambda, H(\lambda)), T_{4}(H)$

$=\bigcap_{\lambda \in[0,1]} f_{4}(\lambda, H(\lambda))$. 则

(1) $T_{3}(H)=T_{4}(H)$

(2) $T_{3}(H)^{\lambda}=\bigcap_{\alpha<\lambda} H(\alpha)$, $T_{3}(H) \underline{\underline{\lambda}}=\bigcup_{\alpha>\lambda} H(\alpha)$.

(3) $T_{3}\left(T_{4}\right)$ 是满射且 $T_{3}\left(\bigcup_{t \in T} H_{t}\right)=\bigcap_{t \in T} T_{3}\left(H_{t}\right)$; $T_{3}\left(\bigcap_{t \in T} H_{t}\right)=\bigcup_{t \in T} T_{3}\left(H_{t}\right) ; T_{3}\left(H^{c}\right)=\left(T_{3}(H)\right)^{c}$.

证明 (1) 设 $A=T_{3}(H)$. 我们先证 $A^{\underline{\lambda}} \subset$ $H(\lambda) \subset A_{\lambda}$. 事实上, 由于

$T_{3}(H)(x)=(\bigvee\{(1-\alpha, \alpha) \mid \alpha \in[0,1]$ 且 $H(\alpha)(x)=$ $0\}) \bigvee\left(\bigvee\left\{(0, \alpha) \mid \alpha \in[0,1]\right.\right.$ 且 $\left.\left.H(\alpha)(x)=\frac{1}{2}\right\}\right)$ $=(\bigvee\{1-\alpha \mid \alpha \in[0,1]$ 且 $H(\alpha)(x)=0\}, \bigwedge\{\alpha \mid \alpha \in[0,1]$ 且 $\left.\left.H(\alpha)(x) \leqslant \frac{1}{2}\right\}\right)$.

因此 $\mu_{A}(x)=\bigvee\{1-\alpha \mid \alpha \in[0,1]$ 且 $H(\alpha)(x)=$ $0\}, \nu_{A}(x)=\bigwedge\left\{\alpha \mid \alpha \in[0,1]\right.$ 且 $\left.H(\alpha)(x) \leqslant \frac{1}{2}\right\}$.

当 $H(\lambda)(x)=0$ 时, $\mu_{A}(x)=\bigvee\{1-\alpha \mid \alpha \in[0,1]$ 且 $H(\alpha)(x)=0\} \geqslant \bigvee\{1-\alpha \mid \alpha \in[0,1]$ 且 $\alpha \geqslant \lambda\}=1-\lambda$, 则 $A \underline{\lambda}(x)=0$. 
当 $A^{\lambda}(x)=1$ 时, $\nu_{A}(x)=\bigwedge\{\alpha \mid \alpha \in[0,1]$ 且 $\left.H(\alpha)(x) \leqslant \frac{1}{2}\right\}>\lambda$, 则 $H(\lambda)(x)=1$.

由于 $A^{\underline{\lambda}}, H(\lambda) \in 3^{X}$, 所以 $A^{\underline{\lambda}} \subset H(\lambda)$.

另一方面, 当 $A^{\lambda}(x)=0$ 时, $\mu_{A}(x)>1-\lambda$. 若 $H(\lambda)(x)>0$, 则 $H(\alpha)(x)>0, \forall \alpha<\lambda$. 于是 $\mu_{A}(x)=\bigvee\{1-\alpha \mid \alpha \in[0,1]$ 且 $H(\alpha)(x)=0\} \leqslant$ $\bigvee\{1-\alpha \mid \alpha>\lambda\}=1-\lambda$. 这与 $\mu_{A}(x)>1-\lambda$ 矛 盾. 因此 $A^{\lambda}(x)=0$ 时, 一定有 $H(\lambda)(x)=0$.

当 $H(\lambda)(x)=1$ 时, 则 $H(\alpha)(x)=1, \forall \alpha<\lambda$, 于 是 $\nu_{A}(x)=\bigwedge\left\{\alpha \mid \alpha \in[0,1]\right.$ 且 $\left.H(\alpha)(x) \leqslant \frac{1}{2}\right\} \geqslant \bigwedge\{\alpha \in$ $[0,1] \mid \alpha>\lambda\}=\lambda$. 则 $A^{\lambda}(x)=1$. 由于 $A^{\lambda}, H(\lambda) \in 3^{X}$, 所以 $H(\lambda) \subset A^{\lambda}$.

因此 $A \underline{\lambda} \subset H(\lambda) \subset A^{\lambda}$. 由定理 4.2 知: $T_{3}(H)=$ $T_{4}(H)$.

(2) 由定理 4.2 知: $T_{3}(H)^{\lambda}=\bigcap_{\alpha<\lambda} H(\alpha), T_{3}(H) \underline{\lambda}$ $=\bigcup_{\alpha>\lambda} H(\alpha)$.

(3) $T_{3}\left(\bigcup_{t \in T} H_{t}\right) \underline{\lambda}=\bigcup_{\alpha>\lambda}\left(\bigcup_{t \in T} H_{t}\right)(\alpha)$

$=\bigcup_{\alpha>\lambda} \bigcup_{t \in T} H_{t}(\alpha)=\bigcup_{t \in T} \bigcup_{\alpha>\lambda} H_{t}(\alpha)$

$=\bigcup_{t \in T} T_{3}\left(H_{t}\right)$.

由性质 3.2(6) 知:

$T_{3}\left(\bigcup_{t \in T} H_{t}\right)^{\underline{\lambda}}=\left(\bigcap_{t \in T} T_{3}\left(H_{t}\right)\right)^{\underline{\lambda}}$.

由定理 4.2(2) 知: $T_{3}\left(\bigcup_{t \in T} H_{t}\right)=\bigcap_{t \in T} T_{3}\left(H_{t}\right)$. 又因为

$T_{3}\left(\bigcap_{t \in T} H_{t}\right)^{\lambda}=\bigcap_{\alpha<\lambda}\left(\bigcap_{t \in T} H_{t}\right)(\alpha)$

$=\bigcap_{\alpha<\lambda} \bigcap_{t \in T} H_{t}(\alpha)=\bigcap_{t \in T} \bigcap_{\alpha<\lambda} H_{t}(\alpha)$

$=\bigcap_{t \in T}\left(T_{3}\left(H_{t}\right)\right)^{\lambda}=\left(\bigcup_{t \in T} T_{1}\left(H_{t}\right)\right)^{\lambda}$.

所以由定理 $4.2(1)$ 知: $T_{3}\left(\bigcap_{t \in T} H_{t}\right)=\bigcup_{t \in T} T_{3}\left(H_{t}\right)$.

类似的,

$T_{3}\left(H^{c}\right)^{\lambda}=\bigcap_{\alpha<\lambda} H^{c}(\alpha)=\bigcap_{\alpha<\lambda}(H(1-\alpha))^{c}=$ $\bigcup_{1-\alpha>1-\lambda}(H(1-\alpha))^{c}=\left(T_{3}(H) \underline{1-\lambda}\right)^{c}=\left(\left(T_{3}(H)\right)^{c}\right)^{\lambda}$. 由定理 4.2 (1) 知: $T_{3}\left(H^{c}\right)=\left(T_{3}(H)\right)^{c}$.

下面给出三值顺序集合套的定义:

定义 5.2 设 $H:[0,1] \rightarrow 3^{X}$ 为一个映射, 若 $\lambda_{1}<\lambda_{2} \Rightarrow H\left(\lambda_{1}\right) \subset H\left(\lambda_{2}\right)$, 则称 $H$ 为 $X$ 上一个三 值顺序集合套, $X$ 上的所有三值顺序集合套的类记 作 $\mathcal{V}(X)$.

在 $\mathcal{V}(X)$ 中规定:

(a) $H_{1} \subset H_{2} \Leftrightarrow H_{1}(\alpha) \supset H_{2}(\alpha), \forall \alpha \in[0,1]$.

(b) $\bigcup_{t \in T} H_{t}:\left(\bigcup_{t \in T} H_{t}\right)(\alpha)=\bigcap_{t \in T} H_{t}(\alpha)$;

$\bigcap_{t \in T} H_{t}:\left(\bigcap_{t \in T} H_{t}\right)(\alpha)=\bigcup_{t \in T} H_{t}(\alpha)$. (c) $H^{c}: H^{c}(\alpha)=(H(1-\alpha))^{c}$;

$\underline{X}(\alpha) \equiv \emptyset, \underline{\emptyset}(\alpha) \equiv X, \forall \alpha \in[0,1]$.

则 $(\mathcal{V}(X), \bigcup, \bigcap, c, \underline{X}, \underline{\emptyset})$ 为一个 $F$ 格.

利用定理 4.3 和定理 4.4 我们可得下列定理.

定理 5.3 设 $T_{i}: \mathcal{V}(X) \rightarrow L^{X}$ 为映射 $(i=$ $5,6)$, 其中 $T_{5}(H)=\bigcup_{\lambda \in[0,1]} f_{5}(\lambda, H(\lambda)), T_{6}(H)=$ $\bigcap_{\lambda \in[0,1]} f_{6}(\lambda, H(\lambda))$. 则

(1) $T_{5}(H)=T_{6}(H)$;

(2) $T_{5}(H)_{[\lambda]}=\bigcap_{\alpha>\lambda} H(\alpha)$, $T_{5}(H)_{[\underline{\lambda}]}=\bigcup_{\alpha<\lambda} H(\alpha)$.

(3) $T_{5}\left(T_{6}\right)$ 为满射且 $T_{5}\left(\bigcup_{t \in T} H_{t}\right)=\bigcap_{t \in T} T_{5}\left(H_{t}\right)$; $T_{5}\left(\bigcap_{t \in T} H_{t}\right)=\bigcup_{t \in T} T_{5}\left(H_{t}\right) ; T_{5}\left(H^{c}\right)=\left(T_{5}(H)\right)^{c}$.

证明 (1) 设 $A=T_{5}(H)$. 我们先证 $A_{[\underline{ }} \subset$ $H(\lambda) \subset A_{[\lambda]}$. 事实上,

$T_{5}(H)(x)=(\bigvee\{(1-\alpha, \alpha) \mid \alpha \in[0,1]$ 且 $H(\alpha)(x)=$ 1) $\bigvee\left(\bigvee\left\{(0, \alpha) \mid \alpha \in[0,1]\right.\right.$ 且 $\left.\left.H(\alpha)(x)=\frac{1}{2}\right\}\right)$

$=(\bigvee\{1-\alpha \mid \alpha \in[0,1]$ 且 $H(\alpha)(x)=1\}, \bigwedge\{\alpha \mid \alpha \in[0,1]$ 且 $\left.\left.H(\alpha)(x) \geqslant \frac{1}{2}\right\}\right)$.

因此 $\mu_{A}(x)=\bigvee\{1-\alpha \mid \alpha \in[0,1]$ 且 $H(\alpha)(x)=1\}$, $\nu_{A}(x)=\bigwedge\left\{\alpha \mid \alpha \in[0,1]\right.$ 且 $\left.H(\alpha)(x) \geqslant \frac{1}{2}\right\}$.

由定理 5.1 的证明知: $A_{[\underline{\lambda}]} \subset H(\lambda) \subset A_{[\lambda]}$. 再由 定理 4.3 知: $T_{5}(H)=T_{6}(H)$.

(2) 由定理 4.3 知: $T_{5}(H)_{[\lambda]}=\bigcap_{\alpha>\lambda} H(\alpha)$, $T_{5}(H)_{[\underline{\lambda}]}=\bigcup_{\alpha<\lambda} H(\alpha)$.

(3) 由于 $T_{5}\left(\bigcup_{t \in T} H_{t}\right)_{[\lambda]}=\bigcap_{\alpha>\lambda}\left(\bigcup_{t \in T} H_{t}\right)(\alpha)$

$=\bigcap_{\alpha>\lambda} \bigcap_{t \in T} H_{t}(\alpha)=\bigcap_{t \in T} \bigcap_{\alpha>\lambda} H_{t}(\alpha)$

$=\bigcap_{t \in T} T_{5}\left(H_{t}\right)_{[\lambda]}=\left(\bigcap_{t \in T} T_{5}\left(H_{t}\right)\right)_{[\lambda]}$.

由定理 4.3(1) 知: $T_{5}\left(\bigcup_{t \in T} H_{t}\right)=\bigcap_{t \in T} T_{5}\left(H_{t}\right)$.

其他证明类似于定理 5.1 的证明.

定理 5.4 设 $T_{i}: \mathcal{V}(X) \rightarrow L^{X}$ 为映射 $(i=7,8)$, 其中 $T_{7}(H)=\bigcup_{\lambda \in[0,1]} f_{7}(\lambda, H(\lambda)), T_{8}(H)=$ $\bigcap_{\lambda \in[0,1]} f_{8}(\lambda, H(\lambda))$. 则

(1) $T_{7}(H)=T_{8}(H)$;

(2) $T_{7}(H)^{[\lambda]}=\bigcap_{\alpha>\lambda} H(\alpha), T_{7}(H)^{[\underline{\lambda}]}$ $=\bigcup_{\alpha<\lambda} H(\alpha)$.

(3) $T_{7}\left(\bigcup_{t \in T} H_{t}\right)=\bigcup_{t \in T} T_{7}\left(H_{t}\right) ; T_{7}\left(\bigcap_{t \in T} H_{t}\right)=$ $\bigcap_{t \in T} T_{7}\left(H_{t}\right) ; T_{7}\left(H^{c}\right)=\left(T_{7}(H)\right)^{c}$.

证明 (1) 令 $A=T_{7}(H)$. 由于 $T_{7}(H)(x)=(\bigvee\{(\alpha, 1-\alpha) \mid \alpha \in[0,1]$ 且 $H(\alpha)(x)=$ $0\}) \bigvee\left(\bigvee\left\{(0,1-\alpha) \mid \alpha \in[0,1]\right.\right.$ 且 $\left.\left.H(\alpha)(x)=\frac{1}{2}\right\}\right)$ 
$=(\bigvee\{\alpha \mid \alpha \in[0,1]$ 且 $H(\alpha)(x)=0\}, \bigwedge\{1-\alpha \mid \alpha \in[0,1]$ 且 $\left.\left.H(\alpha)(x) \leqslant \frac{1}{2}\right\}\right)$.

所以 $\mu_{A}(x)=\bigvee\{\alpha \mid \alpha \in[0,1]$ 且 $H(\alpha)(x)=0\}$, $\nu_{A}(x)=\bigwedge\left\{1-\alpha \mid \alpha \in[0,1]\right.$ 且 $\left.H(\alpha)(x) \leqslant \frac{1}{2}\right\}$.

其他证明类似于定理 5.2 的证明.

注 5.1 由定理 5.1一定理 5.4 知: 每种截集对 应两种表现定理, 直觉模糊集共有八种表现定理.

\section{6 区间值模糊集的截集、分解定理和表现定} 理

设 $A \in \bar{L}^{X}$ 为区间值模糊集, 即 $A(x)=\left[A^{-}(x)\right.$, $\left.A^{+}(x)\right], \forall x \in X$. 令

$$
\mu_{A}(x)=A^{-}(x), \quad \nu_{A}(x)=1-A^{+}(x),
$$

则 $\left(X, \mu_{A}, \nu_{A}\right)$ 为一个直觉模糊集. 由定义 3.2 - 定 义 3.5 我们不难给出下列定义:

\section{定义 6.1}

(1) 称

$$
\begin{aligned}
& A_{\lambda}(x)= \begin{cases}1, & A^{-}(x) \geqslant \lambda ; \\
\frac{1}{2}, & A^{-}(x)<\lambda \leqslant A^{+}(x) ; \\
0, & \lambda>A^{+}(x),\end{cases} \\
& A_{\underline{\underline{\lambda}}}(x)=\left\{\begin{array}{cl}
1, & A^{-}(x)>\lambda ; \\
\frac{1}{2}, & A^{-}(x) \leqslant \lambda<A^{+}(x) ; \\
0, & \lambda \geqslant A^{+}(x) .
\end{array}\right.
\end{aligned}
$$

分别为 $A$ 的 $\lambda$ - 上截集和 $\lambda$ - 强上截集.

(2) 称

$$
\begin{aligned}
& A^{\lambda}(x)= \begin{cases}1, & A^{+}(x) \leqslant \lambda \\
\frac{1}{2}, & A^{-}(x) \leqslant \lambda<A^{+}(x) ; \\
0, & A^{-}(x)>\lambda ;\end{cases} \\
& A^{\underline{\lambda}}(x)=\left\{\begin{array}{cl}
1, & A^{+}(x)<\lambda \\
\frac{1}{2}, & A^{-}(x)<\lambda \leqslant A^{+}(x) ; \\
0, & A^{-}(x) \geqslant \lambda ;
\end{array}\right.
\end{aligned}
$$

分别为 $A$ 的 $\lambda$-下截集和 $\lambda$ - 强下截集.
(3) 称

$$
\begin{aligned}
& A_{[\lambda]}(x)=\left\{\begin{array}{cc}
1, & \lambda+A^{-}(x) \geqslant 1 ; \\
\frac{1}{2}, & A^{-}(x)<1-\lambda \leqslant A^{+}(x) ; \\
0, & \lambda+A^{+}(x)<1,
\end{array}\right. \\
& A_{\underline{\underline{\lambda}}}(x)=\left\{\begin{array}{cc}
1, & \lambda+A^{-}(x)>1 ; \\
\frac{1}{2}, & A^{-}(x) \leqslant 1-\lambda<A^{+}(x) ; \\
0, & \lambda+A^{+}(x) \leqslant 1,
\end{array}\right.
\end{aligned}
$$

分别为 $A$ 的 $\lambda$-上重截集和 $\lambda$-强重上截集.

(4) 称

$$
\begin{aligned}
& A^{[\lambda]}(x)= \begin{cases}1, & \lambda+A^{+}(x) \leqslant 1 ; \\
\frac{1}{2}, & A^{-}(x) \leqslant 1-\lambda<A^{+}(x) ; \\
0, & \lambda+A^{-}(x)>1,\end{cases} \\
& A^{[\underline{\lambda}]}(x)= \begin{cases}1, & \lambda+A^{+}(x)<1 ; \\
\frac{1}{2}, & A^{-}(x)<1-\lambda \leqslant A^{+}(x) ; \\
0, & \lambda+A^{-}(x) \geqslant 1,\end{cases}
\end{aligned}
$$

分别为 $A$ 的 $\lambda$-下重截集和 $\lambda$-强下重截集.

区间值模糊集的截集与直觉模糊集截集有完全 相同的性质, 利用性质 3.1一性质 3.4, 定理 4.1一定 理 4.4 和定理 5.1- 定理 5.4 的结论, 很容易得到区 间值模糊集的分解定理和表现定理.

定义 6.2 设 $g_{i}:[0,1] \times 3^{X} \rightarrow \bar{L}^{X},(\lambda, A) \mapsto$ $g_{i}(\lambda, A)$ 为映射 $(i=1,2, \ldots, 8)$, 其中

$$
\begin{gathered}
g_{1}(\lambda, A)(x)= \begin{cases}{[0,0],} & A(x)=0 ; \\
{[\lambda, \lambda],} & A(x)=1 ; \\
{[0, \lambda],} & A(x)=\frac{1}{2},\end{cases} \\
g_{2}(\lambda, A)(x)= \begin{cases}{[\lambda, \lambda],} & A(x)=0 ; \\
{[1,1],} & A(x)=1 ; \\
{[\lambda, 1],} & A(x)=\frac{1}{2},\end{cases} \\
g_{3}(\lambda, A)(x)= \begin{cases}{[1-\lambda, 1-\lambda],} & A(x)=0 ; \\
{[0,0],} & A(x)=1 ; \\
{[0,1-\lambda],} & A(x)=\frac{1}{2},\end{cases}
\end{gathered}
$$




$$
\begin{gathered}
g_{4}(\lambda, A)(x)= \begin{cases}{[1,1],} & A(x)=0 ; \\
{[1-\lambda, 1-\lambda],} & A(x)=1 ; \\
{[1-\lambda, 1],} & A(x)=\frac{1}{2}\end{cases} \\
g_{5}(\lambda, A)(x)= \begin{cases}{[0,0],} & A(x)=0 ; \\
{[1-\lambda, 1-\lambda],} & A(x)=1 ; \\
{[0,1-\lambda],} & A(x)=\frac{1}{2},\end{cases} \\
g_{6}(\lambda, A)(x)= \begin{cases}{[1-\lambda, 1-\lambda],} & A(x)=0 ; \\
{[1,1],} & A(x)=1 ; \\
{[1-\lambda, 1],} & A(x)=\frac{1}{2},\end{cases} \\
g_{7}(\lambda, A)(x)= \begin{cases}{[\lambda, \lambda],} & A(x)=0 ; \\
{[0,0],} & A(x)=1 ; \\
{[0, \lambda],} & A(x)=\frac{1}{2},\end{cases} \\
g_{8}(\lambda, A)(x)= \begin{cases}{[1,1],} & A(x)=0 ; \\
{[\lambda, \lambda],} & A(x)=1 ; \\
{[\lambda, 1],} & A(x)=\frac{1}{2},\end{cases}
\end{gathered}
$$

则我们有下列定理.

定理 6.1 设 $A$ 为区间值模糊集, 则

(1) $A=\bigcup_{\lambda \in[0,1]} g_{1}\left(\lambda, A_{\lambda}\right)=\bigcap_{\lambda \in[0,1]} g_{2}\left(\lambda, A_{\lambda}\right)$;

(2) $A=\bigcup_{\lambda \in[0,1]} g_{1}\left(\lambda, A_{\underline{\lambda}}\right)=\bigcap_{\lambda \in[0,1]} g_{2}\left(\lambda, A_{\underline{\lambda}}\right)$;

(3) 设映射 $H:[0,1] \rightarrow 3^{X}$ 满足: $A_{\underline{\lambda}} \subset H(\lambda) \subset$ $A_{\lambda}$, 则

(I) $A=\bigcup_{\lambda \in[0,1]} g_{1}(\lambda, H(\lambda))$

$=\bigcap_{\lambda \in[0,1]} g_{2}(\lambda, H(\lambda))$;

(II) $\lambda_{1}<\lambda_{2} \Rightarrow H\left(\lambda_{1}\right) \supset H\left(\lambda_{2}\right)$;

(III) $A_{\lambda}=\bigcap_{\alpha<\lambda} H(\alpha), \quad A_{\underline{\lambda}}=\bigcup_{\alpha>\lambda} H(\alpha)$.

定理 6.2 设 $A$ 为区间值模糊集, 则

(1) $A=\bigcup_{\lambda \in[0,1]} g_{3}\left(\lambda, A^{[\lambda]}\right)=\bigcap_{\lambda \in[0,1]} g_{4}\left(\lambda, A^{[\lambda]}\right)$;

(2) $\left.A=\bigcup_{\lambda \in[0,1]} g_{3}\left(\lambda, A^{[\underline{ }} \underline{ }\right]\right)=\bigcap_{\lambda \in[0,1]} g_{4}\left(\lambda, A^{[\underline{ }} \underline{ }\right)$;

(3) 设映射 $H:[0,1] \rightarrow 3^{X}$ 满足: $A^{[\lambda]} \subset H(\lambda) \subset$ $A^{[\lambda]}$, 则

(I) $A=\bigcup_{\lambda \in[0,1]} g_{3}(\lambda, H(\lambda))$

$=\bigcap_{\lambda \in[0,1]} g_{4}(\lambda, H(\lambda))$;

(II) $\lambda_{1}<\lambda_{2} \Rightarrow H\left(\lambda_{1}\right) \supset H\left(\lambda_{2}\right)$;

(III) $A^{[\lambda]}=\bigcap_{\alpha<\lambda} H(\alpha), \quad A^{[\underline{\lambda}]}=\bigcup_{\alpha>\lambda} H(\alpha)$.

定理 6.3 设 $A$ 为区间值模糊集, 则

(1) $A=\bigcup_{\lambda \in[0,1]} g_{5}\left(\lambda, A_{[\lambda]}\right)=\bigcap_{\lambda \in[0,1]} g_{6}\left(\lambda, A_{[\lambda]}\right)$;

(2) $A=\bigcup_{\lambda \in[0,1]} g_{5}\left(\lambda, A_{[\underline{\lambda}]}\right)=\bigcap_{\lambda \in[0,1]} g_{6}\left(\lambda, A_{[\underline{\lambda}]}\right)$;
(3) 设映射 $H:[0,1] \rightarrow 3^{X}$ 满足: $A_{[\underline{ }]} \subset H(\lambda) \subset$ $A_{[\lambda]}$, 则

(I) $A=\bigcup_{\lambda \in[0,1]} g_{5}(\lambda, H(\lambda))$

$=\bigcap_{\lambda \in[0,1]} g_{6}(\lambda, H(\lambda))$;

(II) $\lambda_{1}<\lambda_{2} \Rightarrow H\left(\lambda_{1}\right) \subset H\left(\lambda_{2}\right)$;

(III) $A_{[\lambda]}=\bigcap_{\alpha>\lambda} H(\alpha), A_{[\underline{\lambda}]}=\bigcup_{\alpha<\lambda} H(\alpha)$.

定理 6.4 设 $A$ 为区间值模糊集, 则

(1) $A=\bigcup_{\lambda \in[0,1]} g_{7}\left(\lambda, A^{\lambda}\right)=\bigcap_{\lambda \in[0,1]} g_{8}\left(\lambda, A^{\lambda}\right)$;

(2) $A=\bigcup_{\lambda \in[0,1]} g_{7}\left(\lambda, A^{\underline{\lambda}}\right)=\bigcap_{\lambda \in[0,1]} g_{8}\left(\lambda, A^{\underline{\lambda}}\right)$;

(3) 设映射 $H:[0,1] \rightarrow 3^{X}$ 满足: $A^{\lambda} \subset H(\lambda) \subset$ $A^{\lambda}$, 则

(I) $A=\bigcup_{\lambda \in[0,1]} g_{7}(\lambda, H(\lambda))$

$=\bigcap_{\lambda \in[0,1]} g_{8}(\lambda, H(\lambda))$;

(II) $\lambda_{1}<\lambda_{2} \Rightarrow H\left(\lambda_{1}\right) \subset H\left(\lambda_{2}\right)$;

(III) $A^{\lambda}=\bigcap_{\alpha>\lambda} H(\alpha), \quad A \underline{\underline{\lambda}}=\bigcup_{\alpha<\lambda} H(\alpha)$.

类似的我们有下列表现定理:

定理 6.5 设 $S_{i}: \mathcal{U}(X) \rightarrow \bar{L}^{X}$ 为映射 $(i=$ $1,2)$, 其中 $S_{1}(H)=\bigcup_{\lambda \in[0,1]} g_{1}(\lambda, H(\lambda)), S_{2}(H)=$ $\bigcap_{\lambda \in[0,1]} g_{2}(\lambda, H(\lambda))$. 则

(1) $S_{1}(H)=S_{2}(H)$;

(2) $S_{1}(H)_{\lambda}=\bigcap_{\alpha<\lambda} H(\alpha)$,

$S_{1}(H)_{\underline{\lambda}}=\bigcup_{\alpha>\lambda} H(\alpha)$.

(3) $S_{1}\left(S_{2}\right)$ 为满射且 $S_{1}\left(\bigcup_{t \in T} H_{t}\right)=\bigcup_{t \in T} S_{1}\left(H_{t}\right)$; $S_{1}\left(\bigcap_{t \in T} H_{t}\right)=\bigcap_{t \in T} S_{1}\left(H_{t}\right) ; S_{1}\left(H^{c}\right)=\left(S_{1}(H)\right)^{c}$.

定理 6.6 设 $S_{i}: \mathcal{U}(X) \rightarrow \bar{L}^{X}$ 为映射 $(i=$ $3,4)$, 其中 $S_{3}(H)=\bigcup_{\lambda \in[0,1]} g_{3}(\lambda, H(\lambda)), S_{4}(H)=$ $\bigcap_{\lambda \in[0,1]} g_{4}(\lambda, H(\lambda))$. 则

(1) $S_{3}(H)=S_{4}(H)$;

(2) $S_{3}(H)^{[\lambda]}=\bigcap_{\alpha<\lambda} H(\alpha)$, $S_{3}(H)^{[\lambda]}=\bigcup_{\alpha>\lambda} H(\alpha)$.

(3) $S_{3}\left(S_{4}\right)$ 是满射且 $S_{3}\left(\bigcup_{t \in T} H_{t}\right)=\bigcap_{t \in T} S_{3}\left(H_{t}\right)$; $S_{3}\left(\bigcap_{t \in T} H_{t}\right)=\bigcup_{t \in T} S_{3}\left(H_{t}\right) ; S_{3}\left(H^{c}\right)=\left(S_{3}(H)\right)^{c}$.

定理 6.7 设 $S_{i}: \mathcal{V}(X) \rightarrow \bar{L}^{X}$ 为映射 $(i=$ $5,6)$, 其中 $S_{5}(H)=\bigcup_{\lambda \in[0,1]} g_{5}(\lambda, H(\lambda)), S_{6}(H)=$ $\bigcap_{\lambda \in[0,1]} g_{6}(\lambda, H(\lambda))$. 则

(1) $S_{5}(H)=S_{6}(H)$;

(2) $S_{5}(H)_{[\lambda]}=\bigcap_{\alpha>\lambda} H(\alpha)$, $S_{5}(H)_{[\underline{\lambda}]}=\bigcup_{\alpha<\lambda} H(\alpha)$.

(3) $S_{5}\left(S_{6}\right)$ 为满射且 $S_{5}\left(\bigcup_{t \in T} H_{t}\right)=\bigcap_{t \in T} S_{5}\left(H_{t}\right)$; $S_{5}\left(\bigcap_{t \in T} H_{t}\right)=\bigcup_{t \in T} S_{5}\left(H_{t}\right) ; S_{5}\left(H^{c}\right)=\left(S_{5}(H)\right)^{c}$. 
定理 6.8 设 $S_{i}: \mathcal{V}(X) \rightarrow \bar{L}^{X}$ 为映射 $(i=$ $7,8)$, 其中 $S_{7}(H)=\bigcup_{\lambda \in[0,1]} g_{7}(\lambda, H(\lambda)), S_{8}(H)=$ $\bigcap_{\lambda \in[0,1]} g_{8}(\lambda, H(\lambda))$. 则

(1) $S_{7}(H)=S_{8}(H)$;

(2) $S_{7}(H)^{\lambda}=\bigcap_{\alpha>\lambda} H(\alpha)$, $S_{7}(H)^{\underline{\lambda}}=\bigcup_{\alpha<\lambda} H(\alpha)$.

(3) $S_{7}\left(\bigcup_{t \in T} H_{t}\right)=\bigcup_{t \in T} S_{7}\left(H_{t}\right) ; S_{7}\left(\bigcap_{t \in T} H_{t}\right)=$ $\bigcap_{t \in T} S_{7}\left(H_{t}\right) ; S_{7}\left(H^{c}\right)=\left(S_{7}(H)\right)^{c}$.

\section{7 结论}

本文给出了直觉模糊集的截集的定义, 讨论了直
觉模糊集的截集的性质, 建立了直觉模糊集的分解定 理和表现定理. 从本文的工作可以看出: (1) 利用本 文已建立的工具, 模糊集的很多理论与应用成果可推 广到直觉模糊集上来; 由于本文的工作实际也建立了 区间值模糊集的截集、分解定理与表现定理, 所以为 研究区间值模糊集提供了有力的工具. (2) 本文的工 作建立了一种新的观点: 模糊集的截集是经典集 (二 值集合), 对应经典集合的幂集为 $2^{X}$; 直觉模糊集的 截集为三值模糊集, 对应的三值模糊集的模糊幂集为 $3^{X}$. 正象 $2^{X}$ 在模糊集研究中的作用一样, $3^{X}$ 在直 觉模糊集和区间值模糊集的研究中也将发挥着重要 的作用.

\section{参考文献}

1 Zadeh L A. Fuzzy sets. Information and Control, 1965, 8(3): 338-353

2 王国俊. $L$-fuzzy 拓扑空间论. 西安: 陕西师范大学出版社, 1988. 1-406

3 Liu Y M, Luo M K. Fuzzy Topology. Singapore: World Scientific Publishing, 1990. 1-306

4 Mordeson J N, Malik D S. Fuzzy Commutative Algebra. Singapore: World Scientific Publishing, 1998. 1-258

5 Mordeson J N, Bhutani K R, Rosenfeld A. Fuzzy Group Theory. New York: Springer, 2005. 1-292

6 张广全. 模糊测度论. 贵阳: 贵州科技出版社, 1994. 1-265

7 吴从炘, 马明. 模糊分析学基础. 北京: 国防工业出版社, 1991. 1-147

8 Bertoluzza C, Solci M, Capodieci M L. Measure of a fuzzy set: the $\alpha$-cut approach in the finite case. Fuzzy Set Syst, 2001, 123(1): 93-102

9 Garcia J N, Kutalik Z, Cho K H, et al. Level sets and minimum volume sets of probability density functions. International J Approx Reason, 2003, 34(1): 25-47

10 Pap E, Surla D. Lebesgue measure of $\alpha$-cuts approach for finding the height of the membership function. Fuzzy Set Sys, 2000, 111(3): 341-350

11 Lai Y J, Hwang C L. Fuzzy Mathematical Programming-Methods and Applications. Berlin: Springer-Verlag, 1992. 1-156

12 徐泽水. 不确定多属性决策方法及应用. 北京: 清华大学出版社, 2004. 3-236

13 Dubois D, Hüllermeier E, Prade H. On the representation of fuzzy rules in terms of crisp rules. Inf Sci, 2003, 151: 301-326

14 Luo C Z, Wang Z P. Representation of compositional relations in fuzzy reasoning. Fuzzy Set Sys, 1990, 36(1): 77-81

15 王国俊. 非经典数理逻辑与近似推理. 北京: 科学出版社, 2000. 24-185

16 罗承忠. 模糊集引论 (上). 北京: 北京师范大学出版社, 1989. 1-486

17 袁学海, 李洪兴, 罗承忠. 几种新的截集及其应用. 模糊系统与数学. 1997, 24(1): 37-43

18 Atanassov K. Intuitionistic fuzzy sets. Fuzzy Set Sys, 1986, 20(1): 87-96

19 Zadeh L A. Outline of a new approach to the analysis of complex systems and decision processes, interval-valued fuzzy sets. IEEE Trans Syst Man Cyber, 1973, 3(1): 28-44

20 Wang G J, He Y Y. Intuitionistic fuzzy sets and L-fuzzy sets. Fuzzy Set Sys, 2000, 110(2): 271-274

21 杨万才, 殷明蛾. 几种模糊集之间的关系. 辽宁师范大学学报 (自然科学版), 2005, 28(2): 143-154

22 李敏. 直觉模糊集的截集. 辽宁师范大学学报 (自然科学版), 2007, 30(2)：152-154

23 曾文艺, 李洪兴, 施显. 区间值模糊集合的分解定理. 北京师范大学学报 (自然科学版), 2003, 39(2): 171-177

24 曾文艺, 李洪兴, 施显. 区间值模糊集合的表现定理. 北京师范大学学报 (自然科学版), 2003, 39(4): 444-447

25 赵宜宾, 曾文艺, 李洪兴. 区间值模糊集合的扩展原理. 北京师范大学学报 (自然科学版), 2007, 43(1): 1-5

26 罗承忠. 模糊集引论 (下). 北京: 北京师范大学出版社, 1991.1-291

27 袁学海, 吴智华. 模糊集截集的公理化描述. 辽宁师范大学学报 (自然科学版), 1999, 22(1): 4-9 